\title{
Can Decarbonylation of Acyl Radicals Be Overcome in Radical Addition Reactions? En Route to a Solution Employing $N$-Acyl Oxazolidinones and $\mathrm{SmI}_{2} / \mathrm{H}_{2} \mathrm{O}$
}

\author{
Christina M. Jensen, Karl B. Lindsay, Rolf H. Taaning, Jakob Karaffa, Anna Mette Hansen \\ and Troels Skrydstrup* \\ Department of Chemistry, University of Aarhus, Langelandsgade 140, 8000 Aarhus C, Denmark
}

\section{Supporting Information}

General Methods. The ${ }^{1} \mathrm{H}$ NMR were recorded at $400 \mathrm{MHz}$ and ${ }^{13} \mathrm{C}$ NMR were recorded at $100 \mathrm{MHz}$. The chemical shifts are reported in ppm downfield to TMS $(\delta=0)$ for ${ }^{1} \mathrm{H}$ NMR and relative to the central $\mathrm{CDCl}_{3}$ resonance $(\delta=77.16)$ for ${ }^{13} \mathrm{C}$ NMR. Solvents were dried according to standard procedures. Flash chromatography was carried out on Merck silica gel 60 (230-400 mesh). All reactions were carried out under an argon atmosphere.

General procedure for the coupling of $\mathbf{N}$-acyl oxazolidinones with acrylamides.

$\mathrm{H}_{2} \mathrm{O}(43 \mu \mathrm{L}, 2.4 \mathrm{mmol})$ was added to a cold solution of the $N$-acyl oxazolidinone $(0.47 \mathrm{mmol})$ and the acrylamide $(0.30$ $\mathrm{mmol})$ in THF $(5.0 \mathrm{~mL})$ at $-78^{\circ} \mathrm{C} . \mathrm{SmI}_{2}(0.1 \mathrm{M}, 14.9 \mathrm{~mL}, 1.5 \mathrm{mmol})$ at room temperature, was added dropwise over $5 \mathrm{~min}$. The solution was left stirring at $-78^{\circ} \mathrm{C}$ for $24 \mathrm{~h}$ unless otherwise stated. Excess $\mathrm{SmI}_{2}$ was oxidized by flushing the mixture with oxygen from a balloon. To the yellow solution was added satd. $\mathrm{NH}_{4} \mathrm{Cl}(3 \mathrm{~mL})$ at $-78^{\circ} \mathrm{C}$ followed by warming to $20^{\circ} \mathrm{C}$. $1 \mathrm{M} \mathrm{HCl}(20 \mathrm{~mL})$ was added followed by extraction with EtOAc $(3 \times 10 \mathrm{~mL})$. The combined organic phases were washed with aqueous $\mathrm{NaS}_{2} \mathrm{O}_{3}(10 \mathrm{~mL})$ and brine $(10 \mathrm{~mL})$, dried over $\mathrm{MgSO}_{4}$ and then evaporated in vacuo. Purification was achieved by flash chromatography on silica gel.

\section{(5S)- $N$-Benzyl 5-benzyloxycarbonylamino-4-oxo-6-phenylhexanamide (2). ${ }^{1}$}

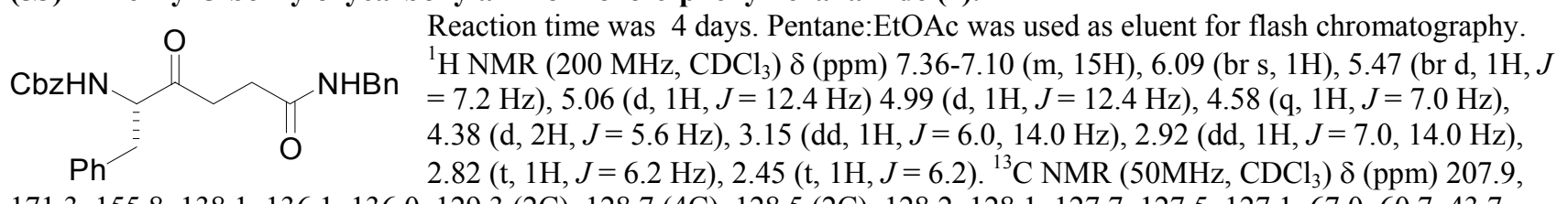

171.3, 155.8, 138.1, 136.1, 136.0, 129.3 (2C), 128.7 (4C), 128.5 (2C), 128.2, 128.1, 127.7, 127.5, 127.1, 67.0, 60.7, 43.7, $37.3,35.4,29.7$.

\section{N-tert-Butyl 4-oxopentanamide.}
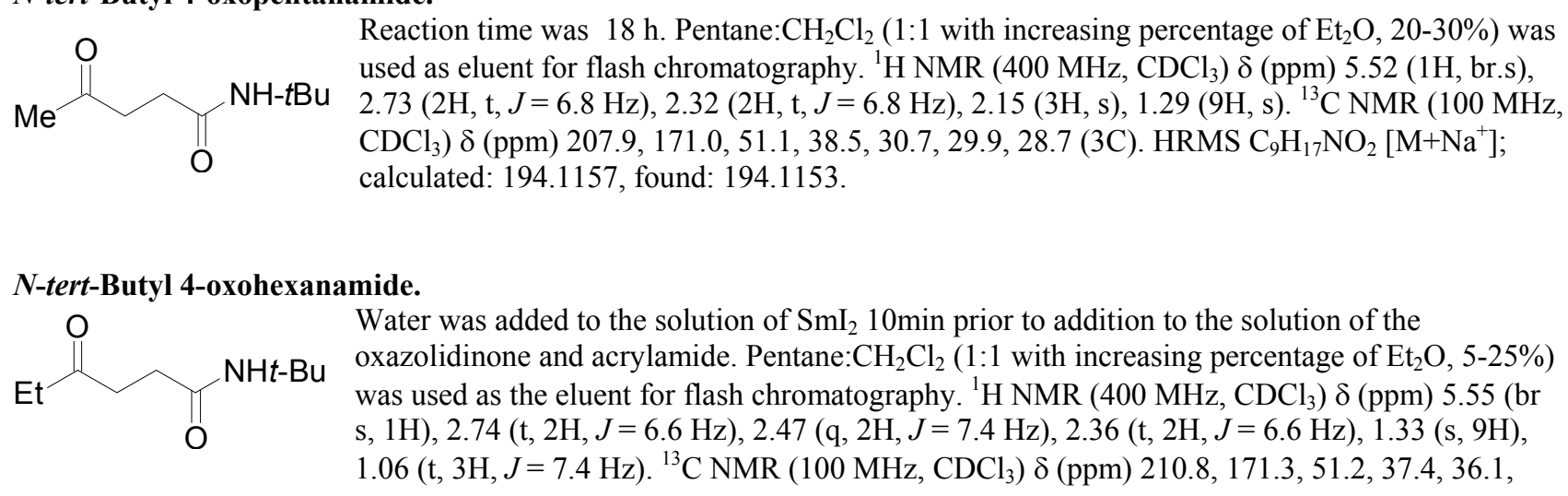
30.9, 28.9 (3C), 7.9. IR 3321, 2975, 1716, 1651, $1549 \mathrm{~cm}^{-1}$. HRMS $\mathrm{C}_{10} \mathrm{H}_{19} \mathrm{NO}_{2}\left[\mathrm{M}+\mathrm{Na}^{+}\right]$; calculated: 208.1308, found: 208.1316 .

\section{N-tert-Butyl 5-methyl-4-oxohexanamide.}

O<smiles>C=O</smiles>

$\mathrm{NH} t-\mathrm{Bu}$

Water was added to the solution of $\mathrm{SmI}_{2} 10 \mathrm{~min}$ prior to addition. $\mathrm{MeOH}: \mathrm{CH}_{2} \mathrm{Cl}_{2}(0.25-1 \%$ $\mathrm{MeOH})$ was used as eluent for flash chromatography. ${ }^{1} \mathrm{H}$ NMR $\left(400 \mathrm{MHz}, \mathrm{CDCl}_{3}\right) \delta(\mathrm{ppm}) 5.47$ (br s, 1H), 2.79 (t, 2H, $J=6.6 \mathrm{~Hz}$ ), 2.63 (septet, $1 \mathrm{H}, J=6.9 \mathrm{~Hz}$ ), 2.35 (t, 2H, $J=6.6 \mathrm{~Hz}), 1.32$ $(\mathrm{s}, 9 \mathrm{H}), 1.11(\mathrm{~d}, 6 \mathrm{H}, J=7.0 \mathrm{~Hz}) .{ }^{13} \mathrm{C} \mathrm{NMR}\left(100 \mathrm{MHz}, \mathrm{CDCl}_{3}\right) \delta(\mathrm{ppm}) 214.1,171.4,51.2,40.9$, $35.7,31.0,28.8$ (3C), 18.3 (2C). IR 3321, 2969, 1711, 1651, $1546 \mathrm{~cm}^{-1}$. HRMS C ${ }_{11} \mathrm{H}_{21} \mathrm{NO}_{2}$

[M+Na ${ }^{+}$; calculated: 222.1465 , found: 222.1476 .

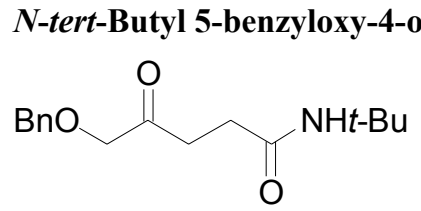

300.1574, found: 300.1570 .

\section{oxopentanamide.}

$\mathrm{CH}_{2} \mathrm{Cl}_{2}: \mathrm{MeOH}(0.5-2 \% \mathrm{MeOH})$ was used as eluent for flash chromatography. ${ }^{1} \mathrm{H}$ NMR (400 $\left.\mathrm{MHz}, \mathrm{CDCl}_{3}\right) \delta(\mathrm{ppm}) 7.40-7.27(\mathrm{~m}, 5 \mathrm{H}), 5.46(\mathrm{br} \mathrm{s}, 1 \mathrm{H}), 4.58(\mathrm{~s}, 1 \mathrm{H}), 4.12(\mathrm{~s}, 1 \mathrm{H}), 2.78(\mathrm{t}$, $2 \mathrm{H}, J=6.3 \mathrm{~Hz}), 2.39(\mathrm{t}, 2 \mathrm{H}, J=6.3 \mathrm{~Hz}), 1.32(\mathrm{~s}, 9 \mathrm{H}) .{ }^{13} \mathrm{C} \mathrm{NMR}\left(100 \mathrm{MHz}, \mathrm{CDCl}_{3}\right) \delta(\mathrm{ppm})$ 208.1, 171.0, 137.4, 128.7 (2C), 128.3 (2C), 128.1, 75.2, 73.6, 51.4, 34.3, 30.5, 29.0 (3C). IR 3294, 3085, 2968, 2919, 1761, $1721 \mathrm{~cm}^{-1}$. HRMS C ${ }_{16} \mathrm{H}_{23} \mathrm{NO}_{3}\left[\mathrm{M}+\mathrm{Na}^{+}\right]$; calculated:

\section{N-tert-Butyl 5-methoxy-4-oxopentanamide.}

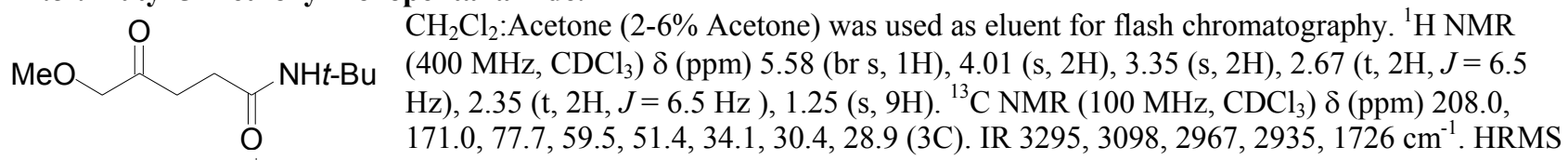

$\mathrm{C}_{10} \mathrm{H}_{19} \mathrm{NO}_{3}\left[\mathrm{M}+\mathrm{Na}^{+}\right]$; calculated: 224.1266 , found: 224.1262 .

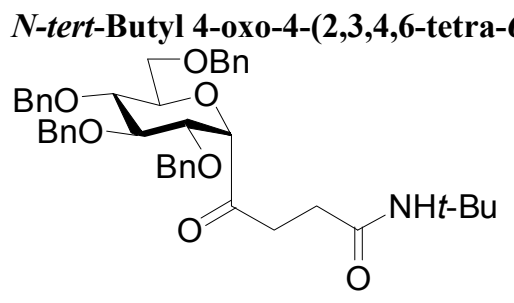

enzyl-a-D-glucopyranosyl)butanamide.

Reaction time was 2 days. The compound showed signs of anomerization over prolonged periods, and hence it was not possible to obtain a pure sample. Pentane: $\mathrm{CH}_{2} \mathrm{Cl}_{2}$ (1:1 with increasing percentage of $\left.\mathrm{Et}_{2} \mathrm{O}, 5-20 \%\right)$ was used as eluent for flash chromatography. ${ }^{1} \mathrm{H} \mathrm{NMR}\left(200 \mathrm{MHz}, \mathrm{CDCl}_{3}\right) \delta(\mathrm{ppm}) 7.21(\mathrm{~m}, 16 \mathrm{H}), 7.09$ $(\mathrm{m}, 4 \mathrm{H}), 5.35(\mathrm{br} \mathrm{s}, 1 \mathrm{H}), 4.65(\mathrm{~d}, 1 \mathrm{H}, J=11.2 \mathrm{~Hz}), 4.61(\mathrm{~d}, 2 \mathrm{H}, J=11.4 \mathrm{~Hz}), 4.61(\mathrm{~d}$, $2 \mathrm{H}, J=11.8 \mathrm{~Hz}), 4.57(\mathrm{~d}, 1 \mathrm{H}, J=11.2 \mathrm{~Hz}), 4.51(\mathrm{~d}, 1 \mathrm{H}, J=12.0 \mathrm{~Hz}), 4.50(\mathrm{~d}, 1 \mathrm{H}, J$ $=11.8 \mathrm{~Hz}), 4.40(\mathrm{~d}, 1 \mathrm{H}, J=12.0 \mathrm{~Hz}), 4.39(\mathrm{~d}, 1 \mathrm{H}, J=11.4 \mathrm{~Hz}), 4.35(\mathrm{~d}, 1 \mathrm{H}, J=4.8)$, $3.99(\mathrm{ddd}, 1 \mathrm{H}, J=9.0, J=3.3, J=3.3 \mathrm{~Hz}), 3.87(\mathrm{t}, 1 \mathrm{H}, J=6.6), 3.82(\mathrm{dd}, 1 \mathrm{H}, J=6.6, J=4.8 \mathrm{~Hz}), 3.60(\mathrm{~m}, 3 \mathrm{H}), 2.84(\mathrm{t}$, $2 \mathrm{H}, J=7.0 \mathrm{~Hz}), 2.23(\mathrm{~m}, 2 \mathrm{H}), 1.24(\mathrm{~s}, 9 \mathrm{H}) . \mathrm{HRMS} \mathrm{C}{ }_{42} \mathrm{H}_{49} \mathrm{NO}_{7}\left[\mathrm{M}+\mathrm{Na}^{+}\right]$; calculated: 702.3407, found: 702.4273.

\section{(5S)- $N$-tert-Butyl 5-benzyloxycarbonylamino-4-oxo-6-phenylhexanamide.}<smiles>O=C(CCC(=O)NC(Cc1ccccc1)C(=O)O)NCc1ccccc1</smiles>
Reaction time was 4 days. $\mathrm{CH}_{2} \mathrm{Cl}_{2}$ :EtOAc $(15: 1)$ was used as eluent for flash chromatography. ${ }^{1} \mathrm{H}$ NMR $\left(400 \mathrm{MHz}, \mathrm{CDCl}_{3}\right) \delta(\mathrm{ppm}) 7.36-7.22(\mathrm{~m}, 8 \mathrm{H}), 7.13(\mathrm{~d}, 2 \mathrm{H}$, $J=6.8 \mathrm{~Hz}), 5.38(\mathrm{~m}, 2 \mathrm{H}), 5.08(\mathrm{~d}, 1 \mathrm{H}, J=12.4 \mathrm{~Hz}), 5.05(\mathrm{~d}, 1 \mathrm{H}, J=12.4 \mathrm{~Hz}), 4.60(\mathrm{q}$, $1 \mathrm{H}, J=6.8 \mathrm{~Hz}), 3.18(\mathrm{dd}, 1 \mathrm{H}, J=14.4,6.0 \mathrm{~Hz}), 2.97(\mathrm{dd}, 1 \mathrm{H}, J=14.4,6.8 \mathrm{~Hz}), 2.88-$ $2.73(\mathrm{~m}, 2 \mathrm{H}), 2.36(\mathrm{t}, 2 \mathrm{H}, J=6.4 \mathrm{~Hz}), 1.32(\mathrm{~s}, 9 \mathrm{H}) .{ }^{13} \mathrm{C} \mathrm{NMR}\left(100 \mathrm{MHz}, \mathrm{CDCl}_{3}\right) \delta$ (ppm) 208.1, 170.7, 155.9, 136.4, 136.1, 129.4 (2C), 128.7 (2C), 128.6 (2C), 128.3, 128.1 (2C), 127.1, 67.0, 60.7, 51.4, 37.3, 35.5, 30.7, 28.9 (3C). IR 3314, 2969, 1697, 1648, $1530 \mathrm{~cm}^{-1}$. HRMS C ${ }_{24} \mathrm{H}_{30} \mathrm{~N}_{2} \mathrm{O}_{4}\left[\mathrm{M}+\mathrm{Na}^{+}\right]$; calculated: 433.2103, found: 433.2104 . 
2-(5-Benzyloxycarbonylamino-4-oxo-6-phenyl-hexanoylamino)-4-methyl-pentanoic acid methyl ester. ${ }^{1}$

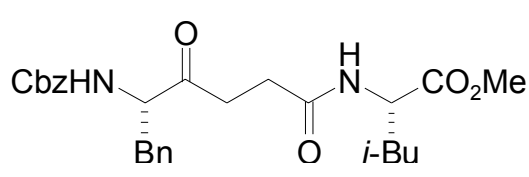

Reaction time was 4 days. ${ }^{1} \mathrm{H}$ NMR $\left(200 \mathrm{MHz}, \mathrm{CDCl}_{3}\right) \delta(\mathrm{ppm}) 7.36-7.22(\mathrm{~m}, 8 \mathrm{H})$, 7.17-7.10 (m, 2H), 6.00 (br d, 1H, $J=8.6 \mathrm{~Hz}), 5.40$ (br d, $1 \mathrm{H}, J=7.2 \mathrm{~Hz}), 5.09$ (d, $1 \mathrm{H}, J=12.2 \mathrm{~Hz}), 5.03(\mathrm{~d}, 1 \mathrm{H}, J=12.2 \mathrm{~Hz}), 4.65-4.54(\mathrm{~m}, 2 \mathrm{H}), 3.71(\mathrm{~s}, 3 \mathrm{H}), 3.18$ $(\mathrm{dd}, 1 \mathrm{H}, J=6.0,14.2 \mathrm{~Hz}) 2.94(\mathrm{dd}, 1 \mathrm{H}, J=7.0,14.2 \mathrm{~Hz}), 2.83(\mathrm{t}, 1 \mathrm{H}, J=6.0 \mathrm{~Hz})$ $2.82(\mathrm{t}, 1 \mathrm{H}, J=6.0 \mathrm{~Hz}), 2.49(\mathrm{t}, 2 \mathrm{H}, J=6.0 \mathrm{~Hz}), 1.72-1.47(\mathrm{~m}, 3 \mathrm{H}), 0,94(\mathrm{~d}, 3 \mathrm{H}, J$ $=6.2 \mathrm{~Hz}), 0.93(\mathrm{~d}, 3 \mathrm{H}, J=6.2 \mathrm{~Hz}) .{ }^{13} \mathrm{C} \mathrm{NMR}\left(50 \mathrm{MHz}, \mathrm{CDCl}_{3}\right) \delta(\mathrm{ppm}) 207.6,173.6,171.3,155.9,136.1,129.4(4 \mathrm{C})$, 128.8 (2C), 128.6, 128.3, 128.2 (2C), 127.2, 67.1, 60.7, 52.4, 50.9, 41.8, 37.4, 35.5, 29.7, 25.0, 22.9, 22.1.

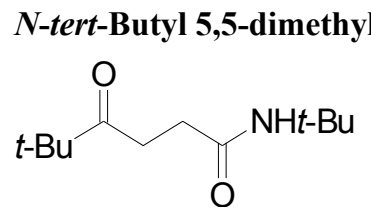

-4-0xohexanamide. Pentane:EtOAc (20-45\% EtOAc) was used as the eluent for flash chromatography. ${ }^{1} \mathrm{H}$ NMR $\left(400 \mathrm{MHz}, \mathrm{CDCl}_{3}\right) \delta(\mathrm{ppm}) 5.45(\mathrm{br} \mathrm{s}, 1 \mathrm{H}), 2.84(\mathrm{t}, 2 \mathrm{H}, J=6.6 \mathrm{~Hz}), 2.33(\mathrm{t}, 2 \mathrm{H}, J=6.6 \mathrm{~Hz})$, $1.32(\mathrm{~s}, 9 \mathrm{H}), 1.15(\mathrm{~s}, 9 \mathrm{H}) .{ }^{13} \mathrm{C} \mathrm{NMR}\left(100 \mathrm{MHz}, \mathrm{CDCl}_{3}\right) \delta(\mathrm{ppm}) 215.5,171.6,51.1,44.0,32.5$, $31.2,28.8$ (3C), 26.6 (3C). IR 3321, 2968, 1706, 1652, $1545 \mathrm{~cm}^{-1}$. HRMS C ${ }_{12} \mathrm{H}_{23} \mathrm{NO}_{2}\left[\mathrm{M}+\mathrm{Na}^{+}\right]$; calculated: 236.1621 , found: 236.1629 .

\section{N-Benzyl 5,5-dimethyl-4-oxohexanamide.

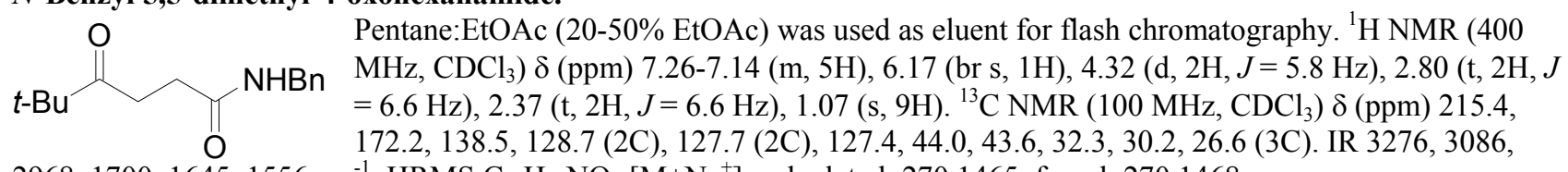
2968, 1700, 1645, $1556 \mathrm{~cm}^{-1}$. HRMS $\mathrm{C}_{15} \mathrm{H}_{21} \mathrm{NO}_{2}\left[\mathrm{M}+\mathrm{Na}^{+}\right]$; calculated: 270.1465, found: 270.1468 .

\section{N-tert-Butyl 4-adamantyl-4-oxobutanamide.

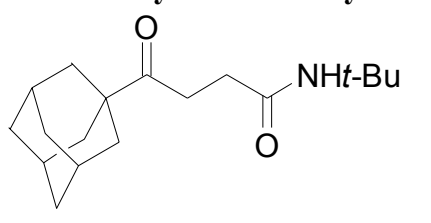 Pentane: $\mathrm{CH}_{2} \mathrm{Cl}_{2}$ (1:1 with increasing percentage of $\left.\mathrm{Et}_{2} \mathrm{O}, 5-20 \%\right)$ was used as eluent for flash chromatography. ${ }^{1} \mathrm{H} \mathrm{NMR}\left(400 \mathrm{MHz}, \mathrm{CDCl}_{3}\right) \delta(\mathrm{ppm}) 5.56(\mathrm{br} \mathrm{s}, 1 \mathrm{H}), 2.75(\mathrm{t}, 2 \mathrm{H}, J=$ $6.6 \mathrm{~Hz}), 2.29$ (t, $J=6.6 \mathrm{~Hz}$ ), 2.00 (br s, 3H), 1.78 (br s, 6H), 1.70 (d, 3H, $J=12.2 \mathrm{~Hz}), 1.64$ $(\mathrm{d}, 3 \mathrm{H}, J=12.2 \mathrm{~Hz}), 1.28(\mathrm{~s}, 9 \mathrm{H}) .{ }^{13} \mathrm{C}$ NMR $\left(100 \mathrm{MHz}, \mathrm{CDCl}_{3}\right) \delta(\mathrm{ppm}) 215.2,171.8,51.2$, 46.4, 38.5 (3C), 36.7(3C), 32.2, 31.1, 29.0(3C), 28.1 (3C). IR 3326, 2960, 2914, 2851, 1691, $1647,1534 \mathrm{~cm}^{-1}$. HRMS C ${ }_{18} \mathrm{H}_{29} \mathrm{NO}_{2}\left[\mathrm{M}+\mathrm{Na}^{+}\right]$; calculated: 314.2096 , found: 314.2102 .}

\section{N-tert-Butyl 4-oxo-5-phenylpentanamide.}



[M+Na ${ }^{+}$; calculated: 270.1465 , found: 270.1468 .

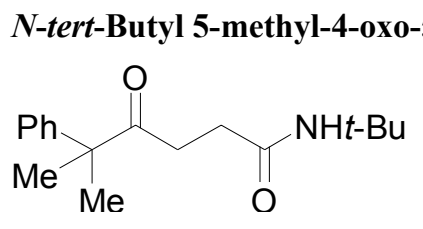

calculated: 298.1783 , found: 298.1788 .

\section{-}

Reaction time was $18 \mathrm{~h}$. Pentane: $\mathrm{CH}_{2} \mathrm{Cl}_{2}$ (1:1 with increasing percentage of $\left.\mathrm{Et}_{2} \mathrm{O}, 5-15 \%\right)$ was used as eluent for flash chromatography. ${ }^{1} \mathrm{H} \mathrm{NMR}\left(400 \mathrm{MHz}, \mathrm{CDCl}_{3}\right) \delta(\mathrm{ppm}) 7.32-$ $7.18(5 \mathrm{H}, \mathrm{m}), 5.55(1 \mathrm{H}$, br. s), $2.54(2 \mathrm{H}, \mathrm{t}, J=6.8 \mathrm{~Hz}), 2.21(2 \mathrm{H}, \mathrm{t}, J=6.8 \mathrm{~Hz}), 1.47(6 \mathrm{H}, \mathrm{s})$, $1.27(9 \mathrm{H}, \mathrm{s}) .{ }^{13} \mathrm{C}$ NMR $\left(100 \mathrm{MHz}, \mathrm{CDCl}_{3}\right) \delta(\mathrm{ppm}) 212.7,171.1,143.8,128.6(2 \mathrm{C}), 126.8$, $125.9(2 \mathrm{C}), 51.8,50.9,33.1,31.3,28.6(3 \mathrm{C}), 25.3(2 \mathrm{C})$. HRMS $\mathrm{C}_{17} \mathrm{H}_{25} \mathrm{NO}_{2}\left[\mathrm{M}+\mathrm{Na}^{+}\right]$; 


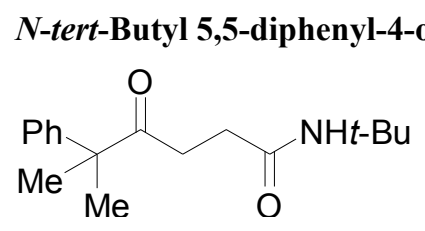

360.1939, found: 360.1939 .

\begin{abstract}
Reaction time was $18 \mathrm{~h}$. Pentane: $\mathrm{CH}_{2} \mathrm{Cl}_{2}\left(1: 1\right.$ with increasing percentage of $\left.\mathrm{Et}_{2} \mathrm{O}, 5-30 \%\right)$ was used as eluent for flash chromatography. ${ }^{1} \mathrm{H}$ NMR $\left(400 \mathrm{MHz}, \mathrm{CDCl}_{3}\right) \delta(\mathrm{ppm}) 7.34-$ $7.13(10 \mathrm{H}, \mathrm{m}), 5.47(1 \mathrm{H}$, br. s), $2.77(2 \mathrm{H}, \mathrm{t}, J=6.8 \mathrm{~Hz}), 2.27(2 \mathrm{H}, \mathrm{t}, J=6.8 \mathrm{~Hz}), 1.31(9 \mathrm{H}$, s), $1.19(3 \mathrm{H}, \mathrm{s}) .{ }^{13} \mathrm{C}$ NMR $\left(100 \mathrm{MHz}, \mathrm{CDCl}_{3}\right) \delta(\mathrm{ppm}) 211.2,171.1,143.7(2 \mathrm{C}), 128.3(8 \mathrm{C})$, 126.8 (2C), 61.6, 51.0, 35.2, 31.5, $28.6(3 \mathrm{C}), 26.3$. HRMS C $\mathrm{C}_{22} \mathrm{H}_{27} \mathrm{NO}_{2}\left[\mathrm{M}+\mathrm{Na}{ }^{+}\right]$; calculated:
\end{abstract}

\title{
General procedure for the coupling of $\mathbf{N}$-acyl oxazolidinones with acrylates.
}

$\mathrm{H}_{2} \mathrm{O}(54 \mu \mathrm{L}, 3 \mathrm{mmol})$ was added to a cold solution of the $N$-acyl oxazolidinone $(0.56 \mathrm{mmol})$ and the acrylate $(1.68 \mathrm{mmol})$ in THF $(10.0 \mathrm{~mL})$ at $-40^{\circ} \mathrm{C}$. A THF solution of $\operatorname{SmI}_{2}(0.1 \mathrm{M}, 14.9 \mathrm{~mL}, 1.5 \mathrm{mmol})$ at $-40^{\circ} \mathrm{C}$, was added and the blue solution was left stirring at $-40^{\circ} \mathrm{C}$ for $24 \mathrm{~h}$. Excess $\mathrm{SmI}_{2}$ was oxidized by flushing the mixture with oxygen from a balloon. To the yellow solution was added satd. $\mathrm{NH}_{4} \mathrm{Cl}(3 \mathrm{~mL})$ at $-40^{\circ} \mathrm{C}$ followed by warming to $20^{\circ} \mathrm{C} .1 \mathrm{M} \mathrm{HCl}(20 \mathrm{~mL})$ was added followed by extraction with EtOAc $(3 \times 10 \mathrm{~mL})$. The combined organic phases were washed with aqueous $\mathrm{NaS}_{2} \mathrm{O}_{3}(10 \mathrm{~mL})$, dried over $\mathrm{MgSO}_{4}$ and then evaporated in vacuo. Purification was achieved by flash chromatography on silica gel.

\section{$N$-Butyl 5-(benzyloxy)-4-oxopentanoate.}

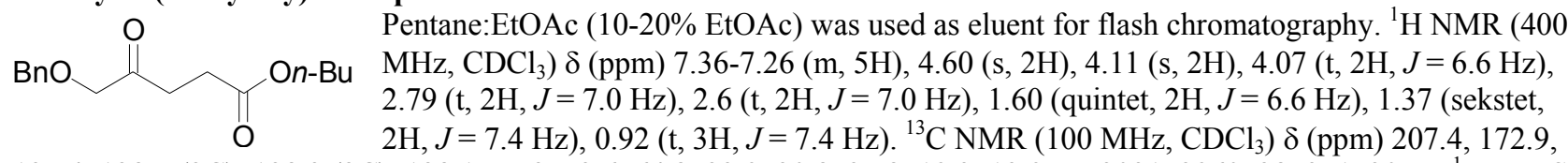
137.4, 128.7 (2C), 128.2 (2C), 128.1, 75.2, 73.6, 64.8, 33.9, 30.8, 27.8, 19.3, 13.9. IR 3031, 2960, 2873, $1730 \mathrm{~cm}^{-1}$. HRMS $\mathrm{C}_{16} \mathrm{H}_{22} \mathrm{O}_{4}\left[\mathrm{M}+\mathrm{Na}^{+}\right]$; calculated: 301.1417, found: 301.1414 . 13.9. IR 2962, 2874, 1736, $1709 \mathrm{~cm}^{-1}$. HRMS $\mathrm{C}_{12} \mathrm{H}_{22} \mathrm{O}_{3}\left[\mathrm{M}+\mathrm{Na}^{+}\right]$; calculated: 237.1013 , found: 237.1021.

\section{$N$-Butyl 4-oxo-5-phenylpentanoate. \\ Pentane:EtOAc (2.5-5\% EtOAc) was used as eluent for flash chromatography. ${ }^{1} \mathrm{H} \mathrm{NMR}(400 \mathrm{MHz}$, $\left.\mathrm{Bn} \quad \mathrm{CDCl}_{3}\right) \delta(\mathrm{ppm}) 7.34-7.20(\mathrm{~m}, 5 \mathrm{H}), 4.05(\mathrm{t}, 2 \mathrm{H}, J=6.4 \mathrm{~Hz}), 3.73(\mathrm{~s}, 2 \mathrm{H}), 2.75(\mathrm{t}, 2 \mathrm{H}, J=7.0 \mathrm{~Hz})$, \\ $\begin{aligned} \mathrm{On}-\mathrm{Bu} & 2.55(\mathrm{t}, 2 \mathrm{H}, J=7.10 \mathrm{~Hz}), 1.58 \text { (quintet, } 2 \mathrm{H}, J=7.0 \mathrm{~Hz}), 1.35(\text { sekstet, } 2 \mathrm{H}, J=7.4 \mathrm{~Hz}), 0.92(\mathrm{t}, \\ \mathrm{O} & 7.4 \mathrm{~Hz}) \cdot{ }^{13} \mathrm{C} \mathrm{NMR}\left(100 \mathrm{MHz}, \mathrm{CDCl}_{3}\right) \delta(\mathrm{ppm}) 206.6,173.0,134.3,129.7(2 \mathrm{C}), 129.0(2 \mathrm{C}) \text {, }\end{aligned}$}

$127.3,64.8,50.3,36.7,30.8,28.3,19.3,13.9$. IR 3030, 2961, 2874, $1733 \mathrm{~cm}^{-1}$. HRMS C${ }_{15} \mathrm{H}_{20} \mathrm{O}_{3}\left[\mathrm{M}^{2} \mathrm{Na}^{+}\right]$; calculated: 271.1314, found: 271.1316 .

\section{4-Cyano-1-phenylbutan-4-one.}

3-(phenylacetyl)-2-oxazolidinone (93 mg, 0,454 mmol) was dissolved in THF (7.5 mL) after which
acrylonitrile $(98 \mu \mathrm{L}, 1.50 \mathrm{mmol})$ and $\mathrm{H}_{2} \mathrm{O}(54 \mu \mathrm{L}, 3.00 \mathrm{mmol})$ were added. The mixture was cooled to -
$\mathrm{Bn}$ mixture was stirred at $-78{ }^{\circ} \mathrm{C}$ for $18 \mathrm{~h}$, and excess $\mathrm{SmI}_{2}$ was oxidized by flushing the mixture with oxygen from a balloon. To the yellow solution was added satd. $\mathrm{NH}_{4} \mathrm{Cl}(3 \mathrm{~mL})$ at $-78^{\circ} \mathrm{C}$ followed by warming to $20^{\circ} \mathrm{C} .1 \mathrm{M} \mathrm{HCl}(20 \mathrm{~mL})$ was added followed by extraction with EtOAc $(3 \times 10 \mathrm{~mL})$. The combined organic phases were washed with aqueous $\mathrm{Na}_{2} \mathrm{~S}_{2} \mathrm{O}_{3}(10$ $\mathrm{mL}$ ), dried over $\mathrm{MgSO}_{4}$ and then evaporated in vacuo. Purification was achieved by flash chromatography on silica gel (pentane:EtOAc 9:1 to 3:2 was used as eluant) which gave the title compound (41 $\mathrm{mg}, 0.237 \mathrm{mmol}, 52 \%$ ) as a colorless oil. 
${ }^{1} \mathrm{H} \mathrm{NMR}\left(400 \mathrm{MHz}, \mathrm{CDCl}_{3}\right) \delta(\mathrm{ppm}) 7.37-7.18(\mathrm{~m}, 5 \mathrm{H}), 3.72(\mathrm{~s}, 2 \mathrm{H}), 2.80$ (d, $\left.2 \mathrm{H}, J=7.2 \mathrm{~Hz}\right), 2.52(\mathrm{t}, 2 \mathrm{H}, J=7.2 \mathrm{~Hz})$.

${ }^{13} \mathrm{C} \mathrm{NMR}\left(100 \mathrm{MHz}, \mathrm{CDCl}_{3}\right) \delta$ (ppm) 203.8, 133.1, 129.3 (2C), 128.9 (2C), 127.4, 118.8, 49.6, 36.8, 11.3. IR 3030, 2921, 2249, 1712, 1603, $1494 \mathrm{~cm}^{-1}$. HRMS $\mathrm{C}_{11} \mathrm{H}_{11} \mathrm{NO}\left[\mathrm{M}+\mathrm{Na}^{+}\right]$; calculated: 196.0738 found: 196.0732.

\section{References}

1. Blakskjær, P.; Høj, B.; Riber, D.; Skrydstrup, T. J. Am. Chem. Soc. 2003, 125, 4030. 

kb1111a13_24

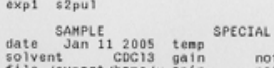

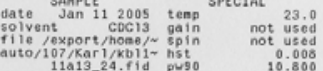

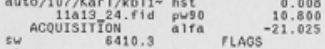

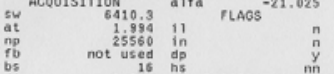

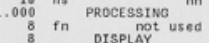

ct TRANSHITter ${ }^{8}$ Sp DISPLAY

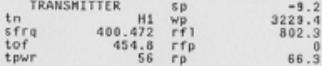

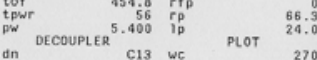

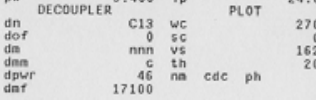

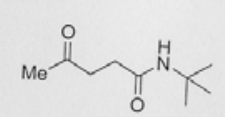

Pulse seavence: sapu

Solvent: coc13 $139.1 \mathrm{~K}$
Temp. $26.0 \mathrm{C}, 29.1 \mathrm{~K}$

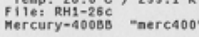

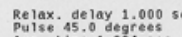

Acg tine 1 , 994 se

OBSERVE H1, 400.4698141 MHZ

FT 5120,32763
Total tine 0 in, 25 sec 


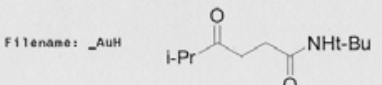

Archive directory:
sample directory:

Pu1se sequence: s2pu1

Solvent: Coc13
Temp. $26.0 \mathrm{C}$ C 299.1

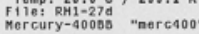

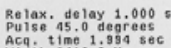

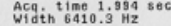

So repetitions
ogsepVE

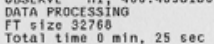

JK290_recol

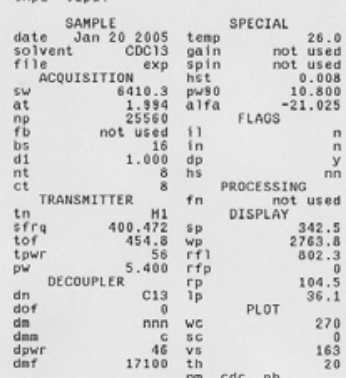

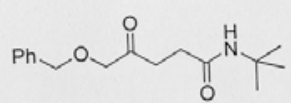

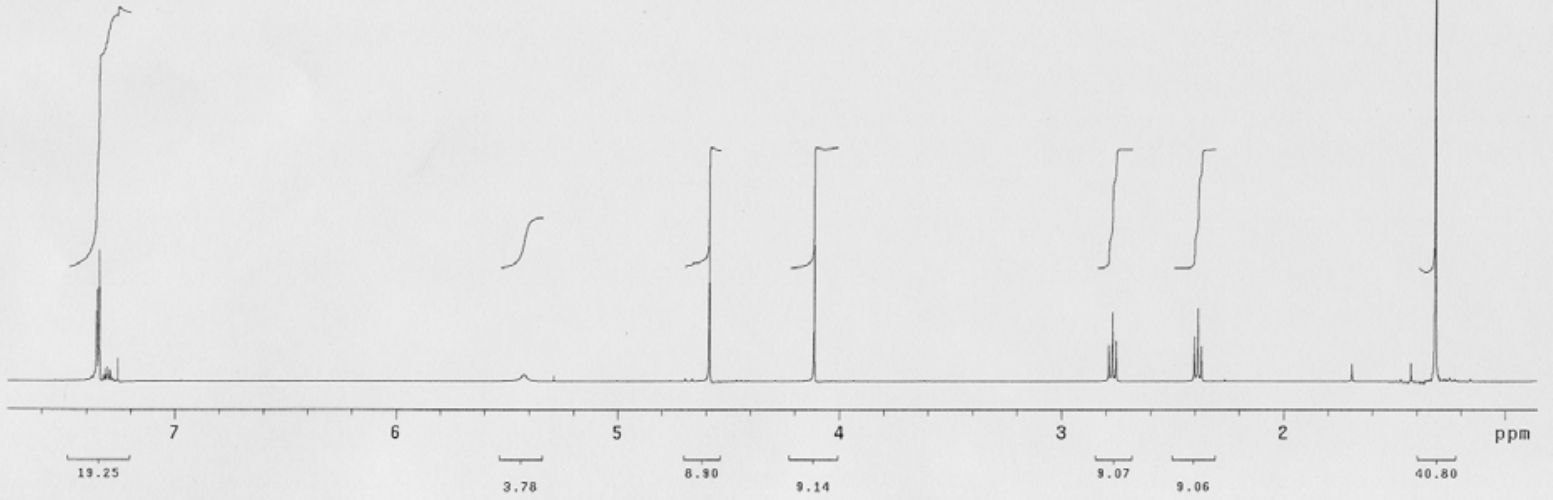




\section{$J k \exp 24 b$
$\operatorname{exp2} 3220 u$}
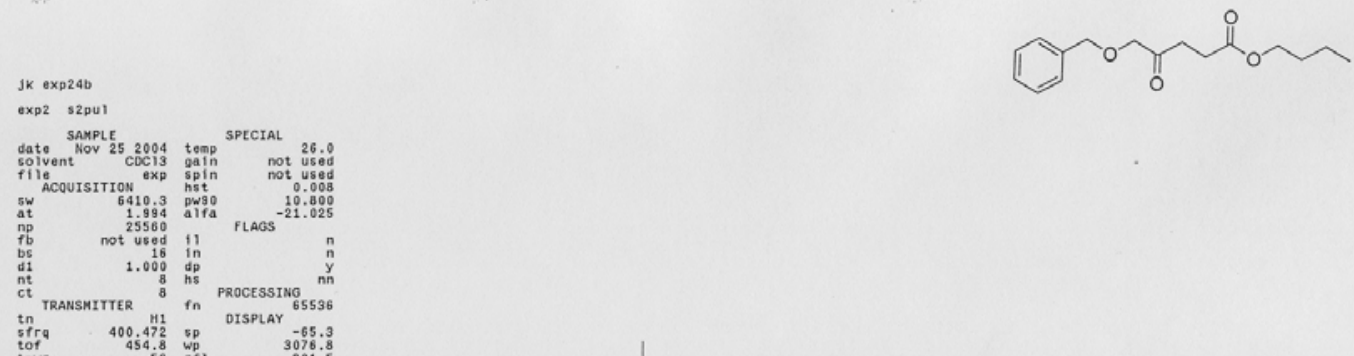

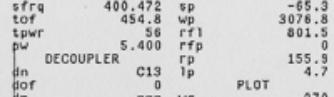

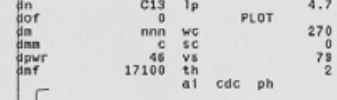

$\int$ 

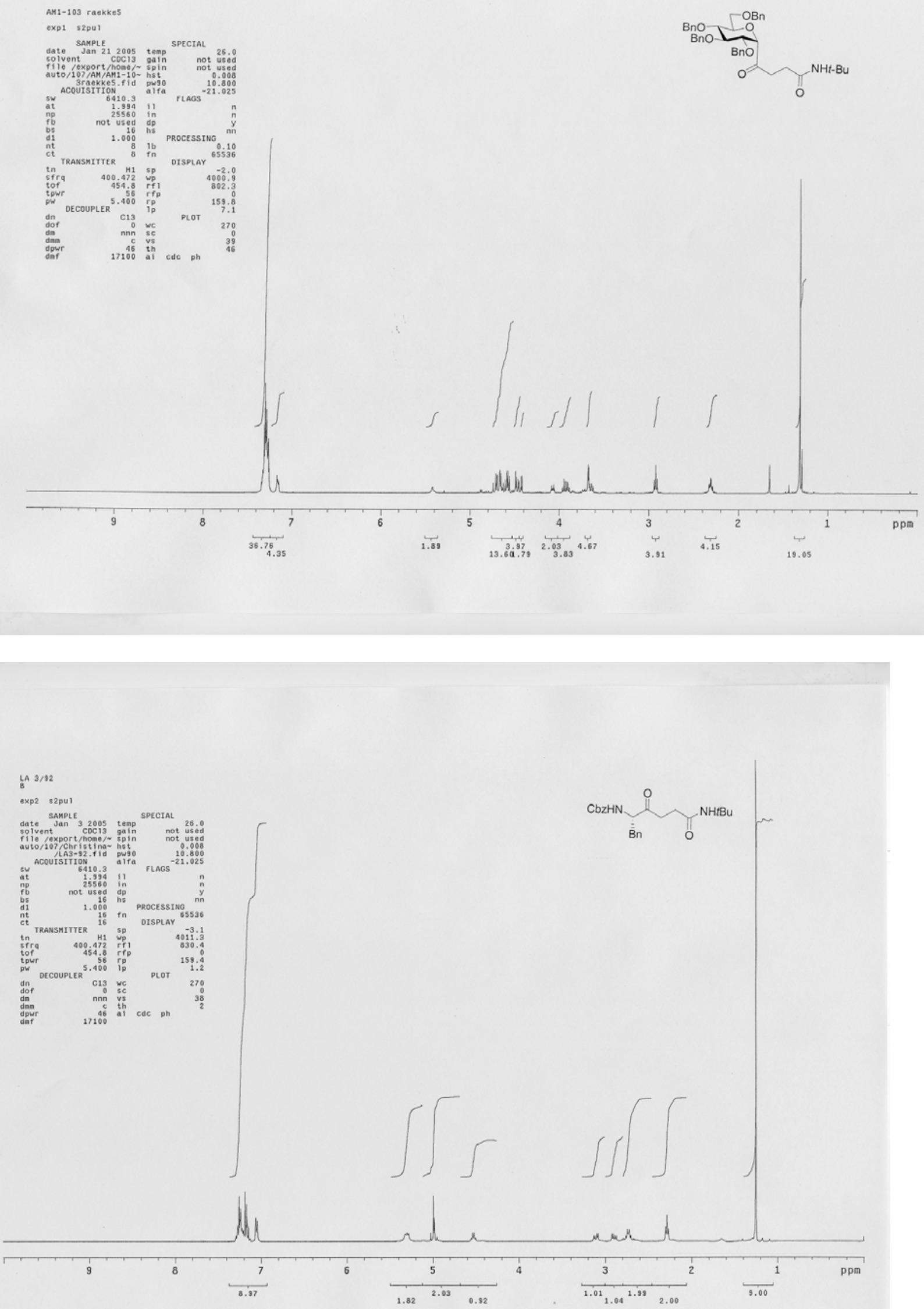
RH1-2b

Archive directory: /export/homo/auto/vnersys/date
Sample directory:

puree sequence: 2 pit

Solvent: coc1s 299.1

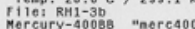

Relax. de lay $1.000 \mathrm{sec}$

Acg. ${ }^{2 i n e} 1.994 \mathrm{sec}$

or repet titions 400.4698517 MHE

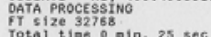
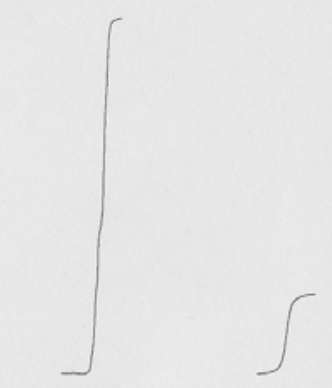

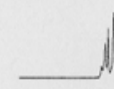
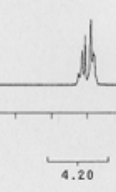

4.20

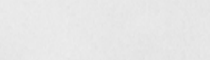

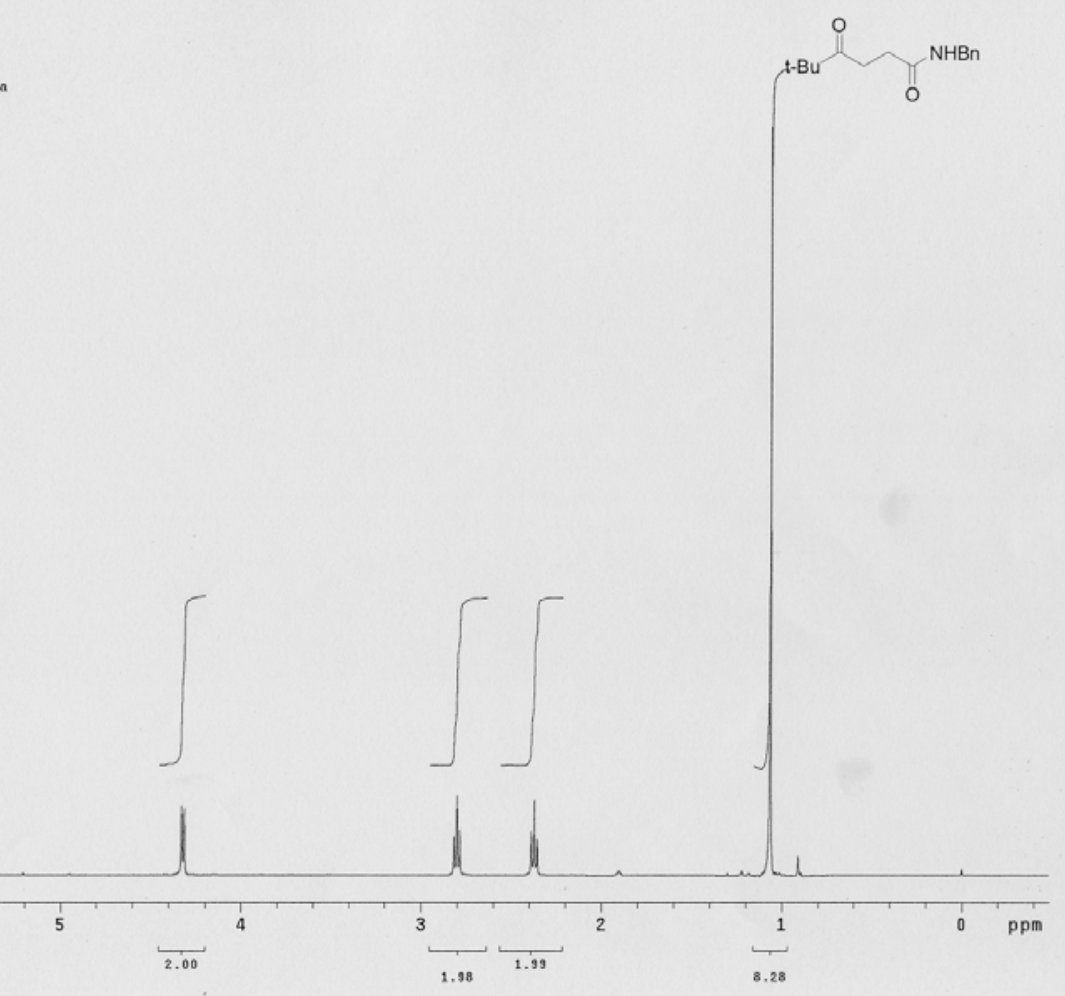

RH1-206

Archive directory: /export/home/auto/vnmrsys/tuata
Sample directory:

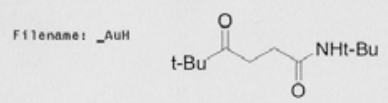

Pulse sequence: s2pul

Solvent: Coc $13,299.1 \mathrm{~K}$
Temp. $26.0 \mathrm{C} / 29.1 \mathrm{x}$

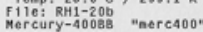

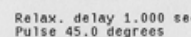

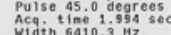

Vidth $6410.3 \mathrm{~Hz}$
8 repetitions
osserve H1. 400.4698254 Me1

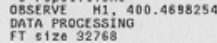

Total time 0 min, $25 \mathrm{sec}$ 
JK exp27b

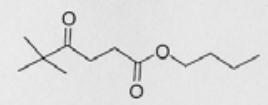

exp1 s2pu 1

date SAMPLE, Nov 2004 tenp SPECIAL
solvent

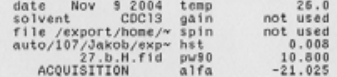

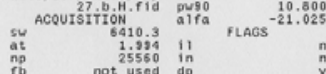

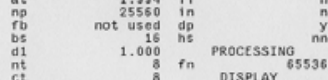

th TRANSMITTER ${ }^{\text {H1 }}$ sp DISPLAY

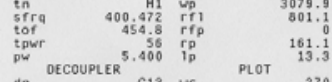

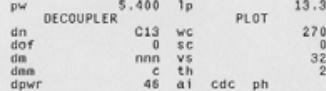

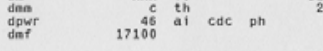

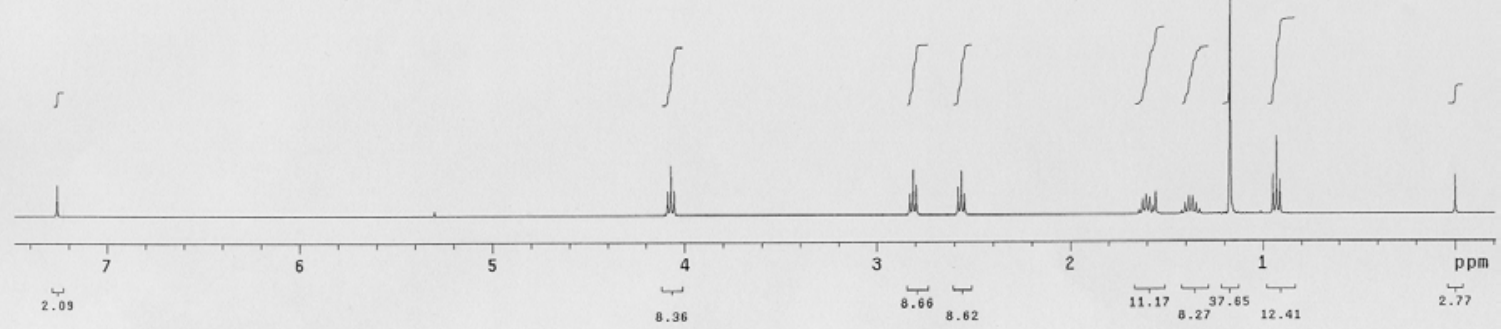

AM1-97

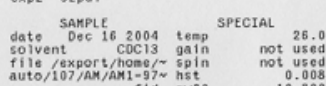

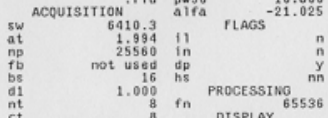

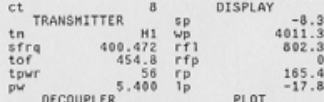

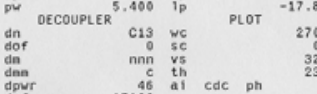

2

diaf $17100^{4}$ ai cde ph 
S13
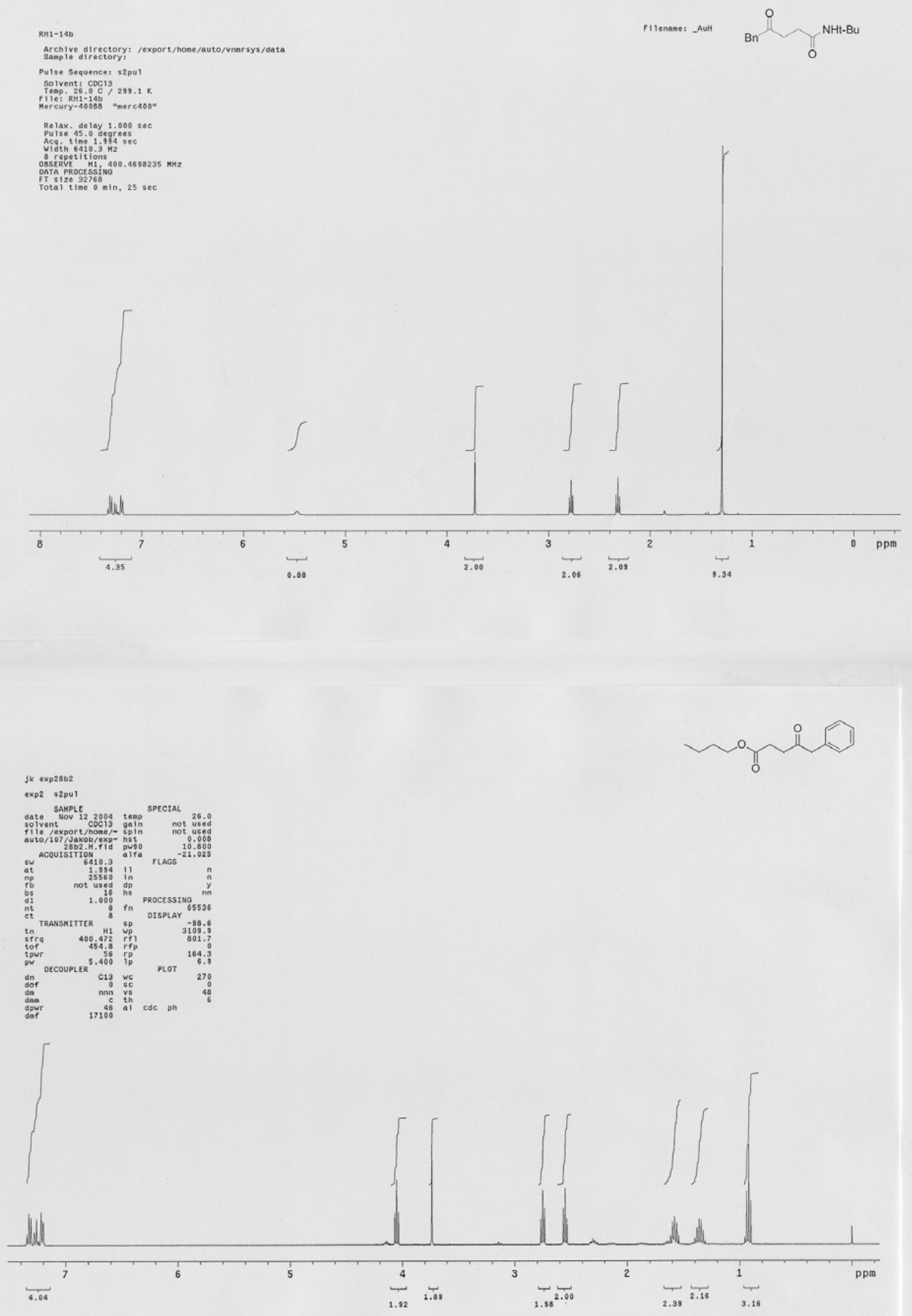
S14

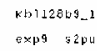

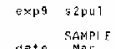

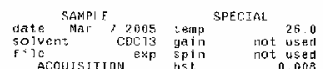

少
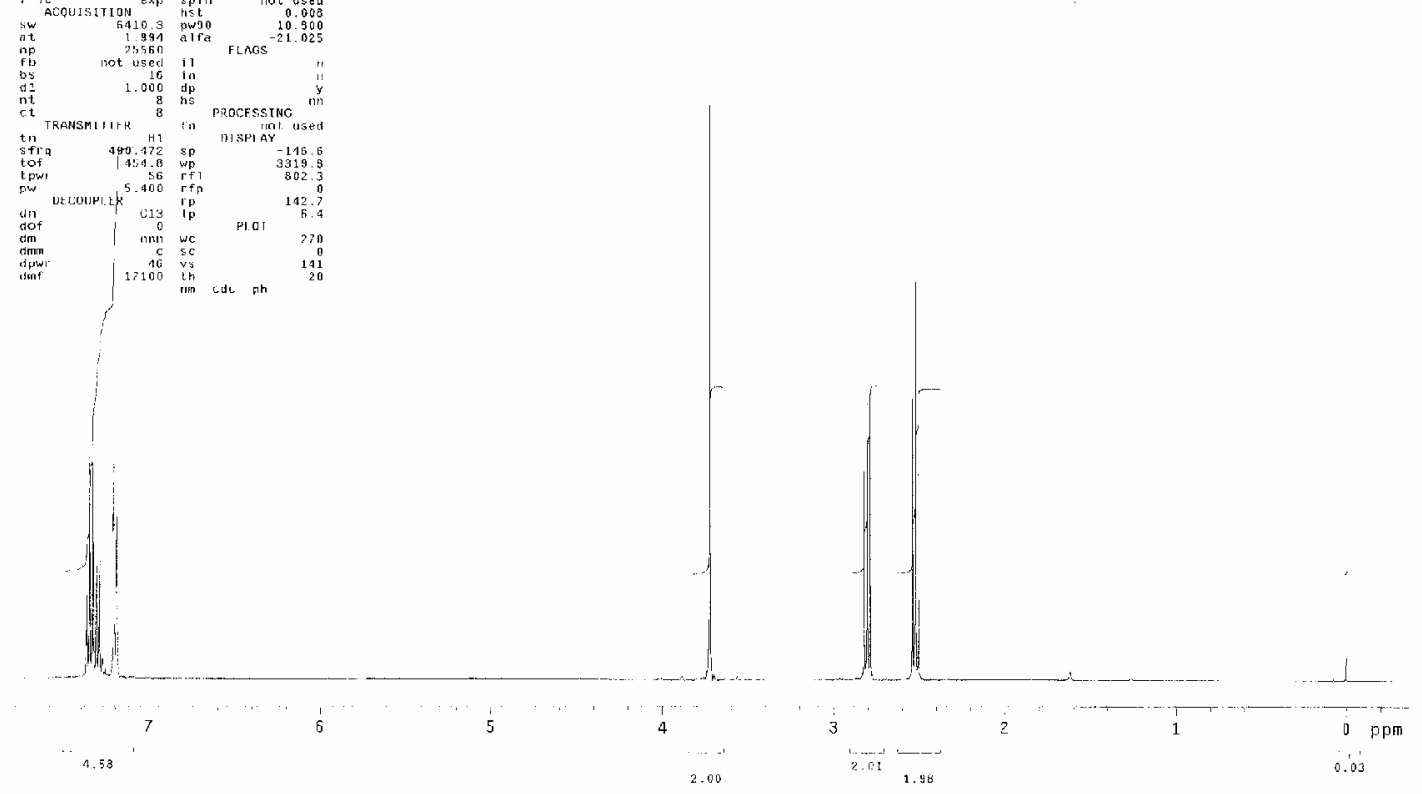

kb1101b_2ndcolfrac12

exp1 $52 p u 1$

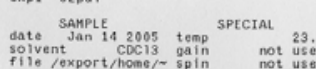

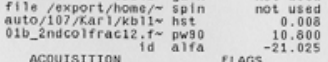

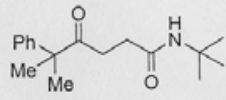

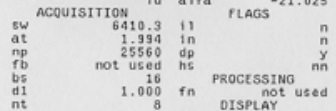

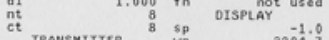

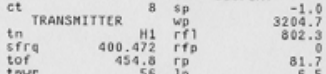

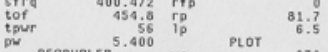

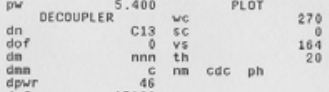

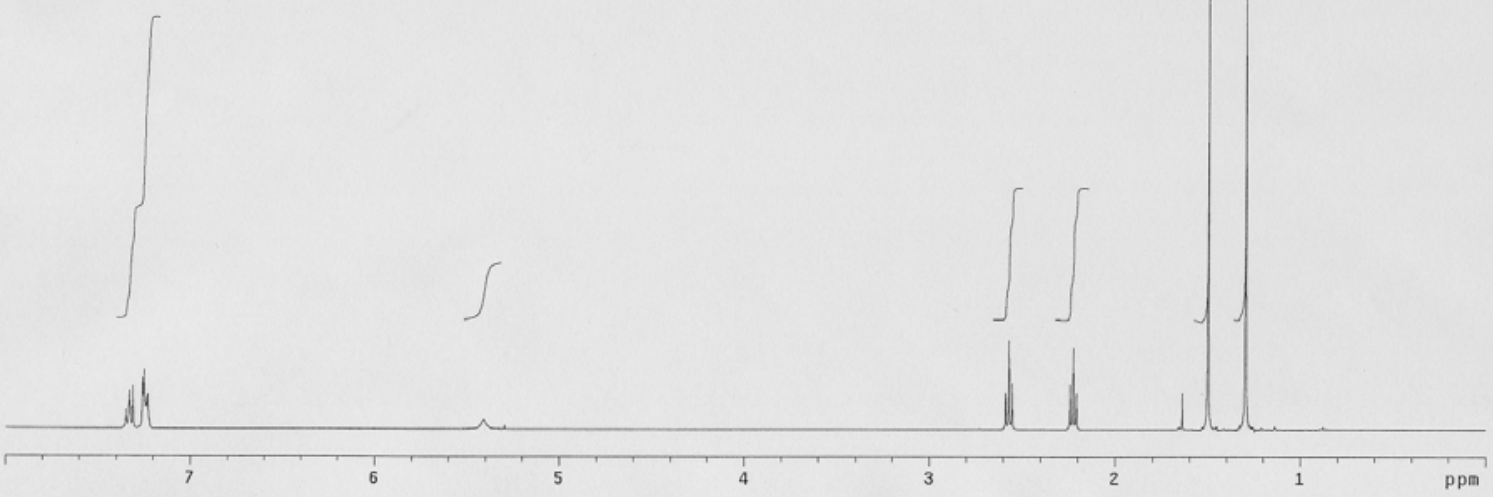




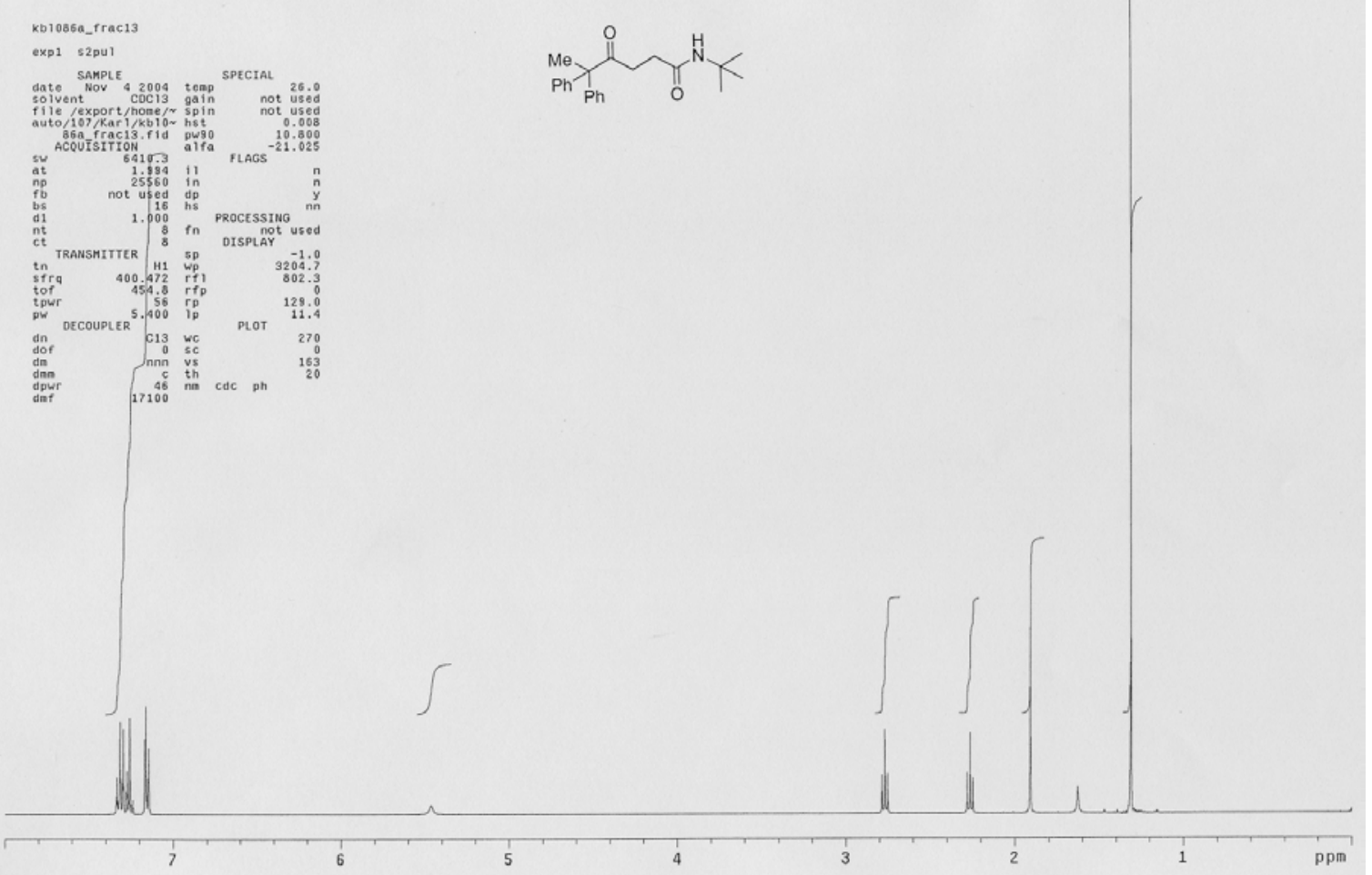


exp2 $25 \mathrm{put}$

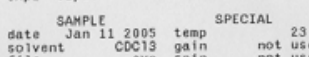

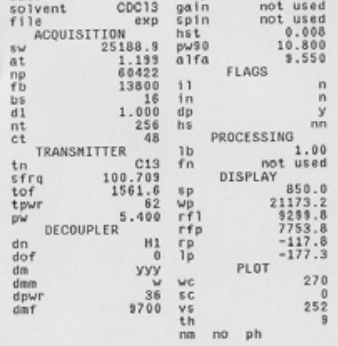

Filenane: AuC
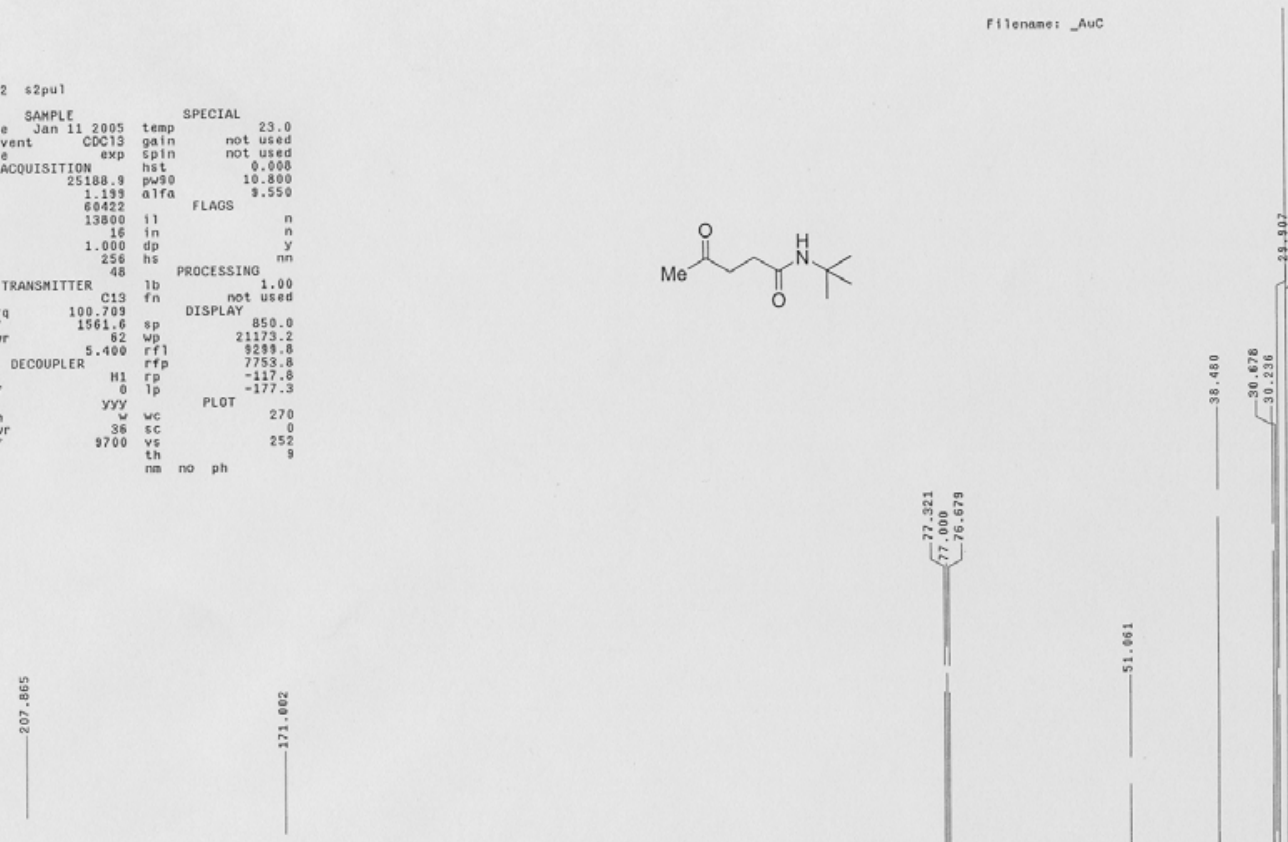

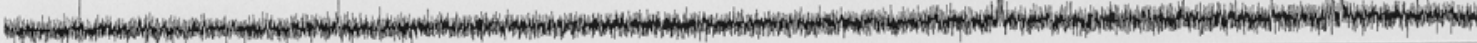
200

RH1-26C13

Archive directory: /export/hone/auto/vnarsys/data
Sample directory:

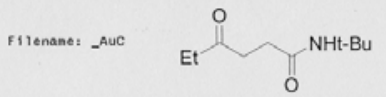

Pulse Sequence: $82 p u l$

Solvent: coc1s $19.1 \%$
Temp. $26.0 \mathrm{C}$. $299.1 \mathrm{~K}$

Mercury-400BB "merc400

Relax. de lay 1.000 sec
Pulse 45.0 degrees

Acq time 1.199 sec

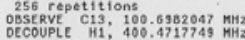

Power $36 \mathrm{HB}, 400$.

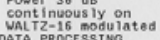

DATA PRoCESSING
Line broadening $1.0 \mathrm{~Hz}$

IT size 55536 ing $1.0 \mathrm{~Hz}$

for 
Relax. delay 1.000
Pulse. 45.0 degrees

Acg tine $1.119 \mathrm{sec}$

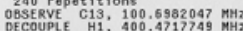

Ower 35 did

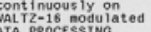

Lin
Line brostenting $1.0 \mathrm{~Hz}$

FT 512065535
Total time 41 nin, $10 \mathrm{sec}$

\section{7}

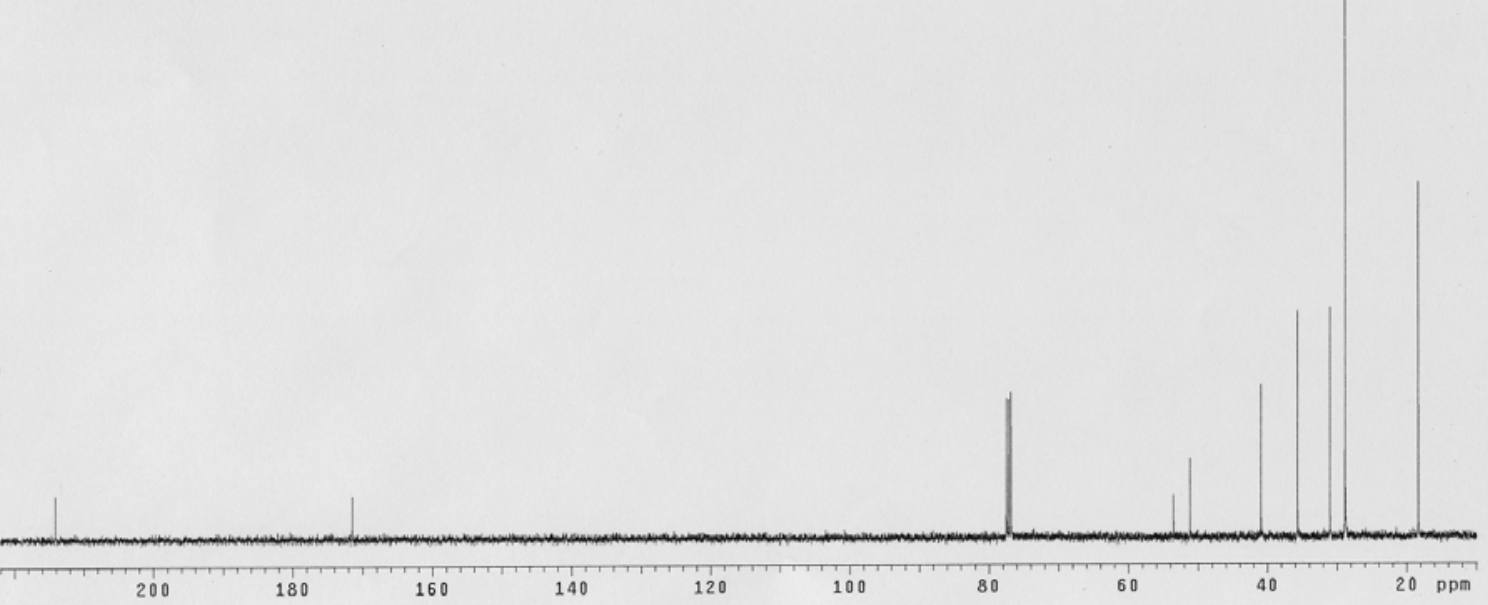

refi

exp1 E2pul

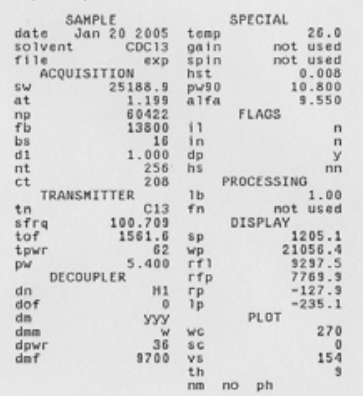
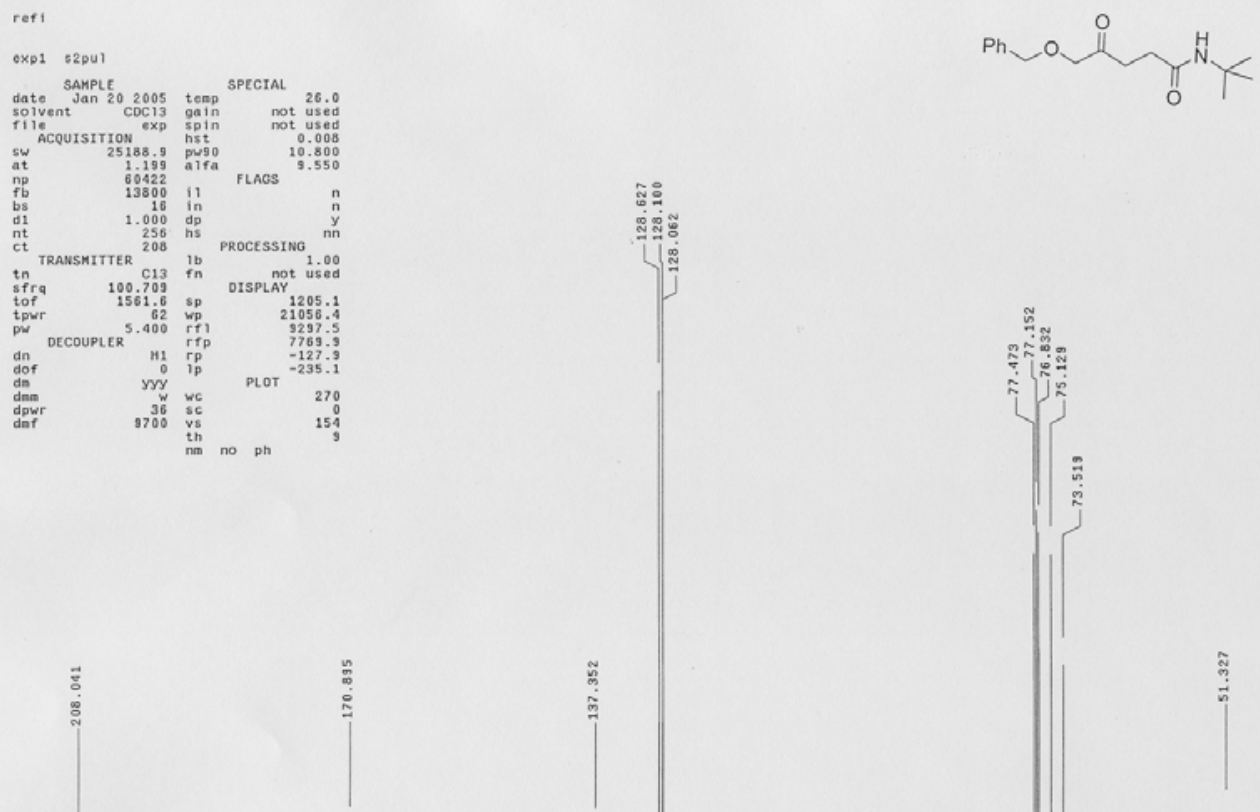

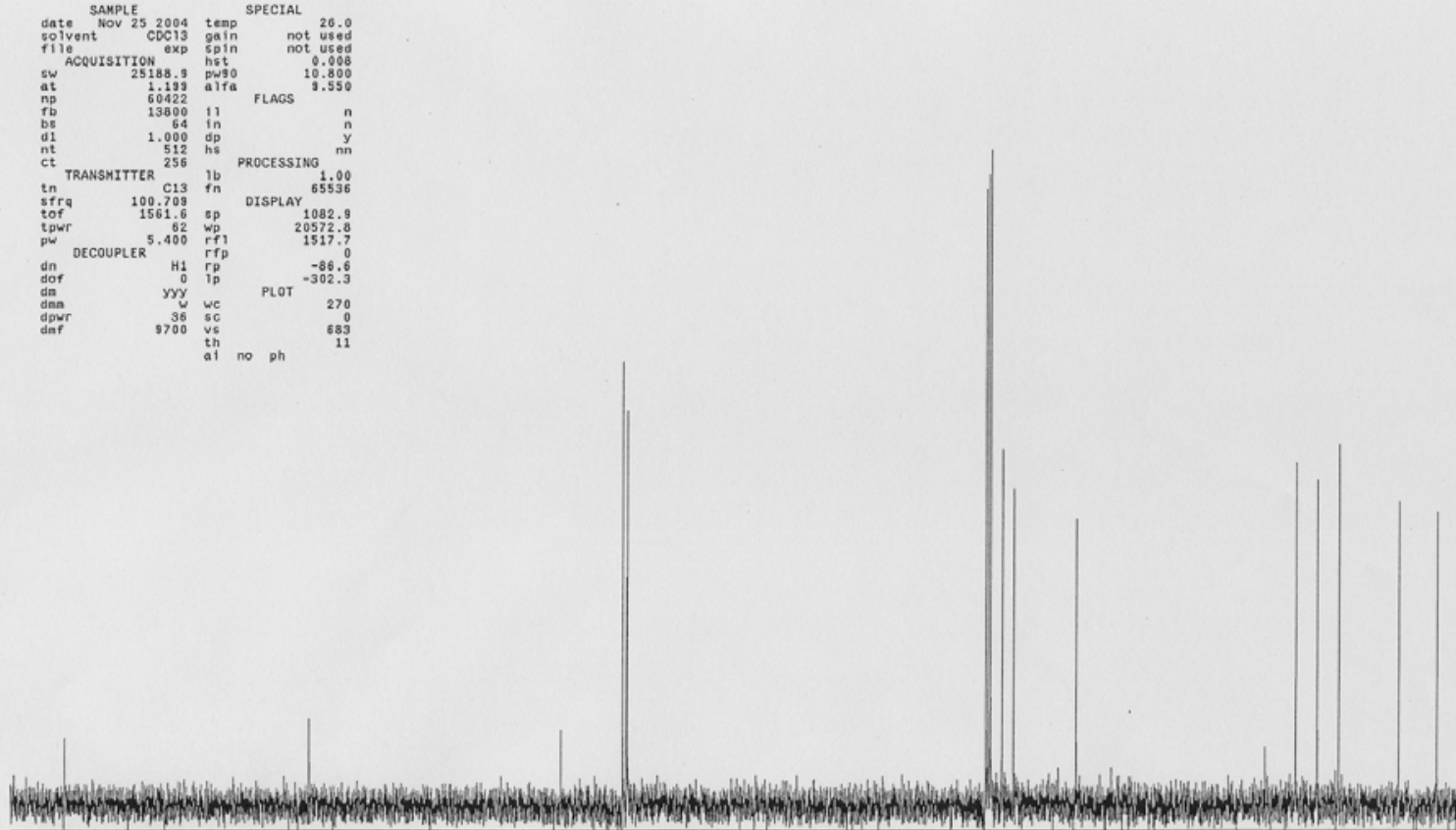

\section{$J K$ exp30b}

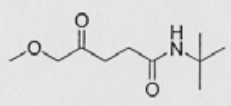

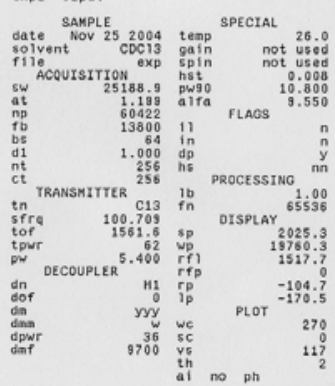

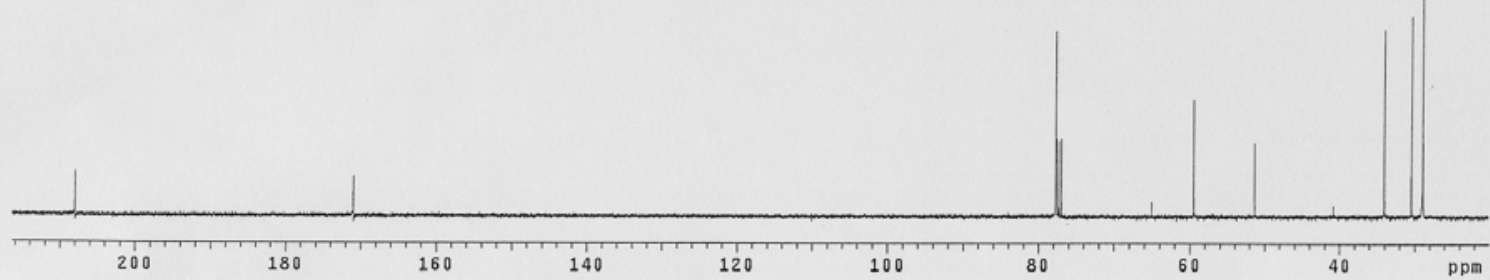


LA3-92-Carbon
QXp2 $\$ 2$ pu 1

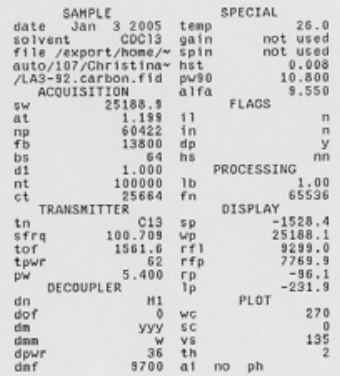

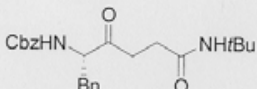

Bn 0

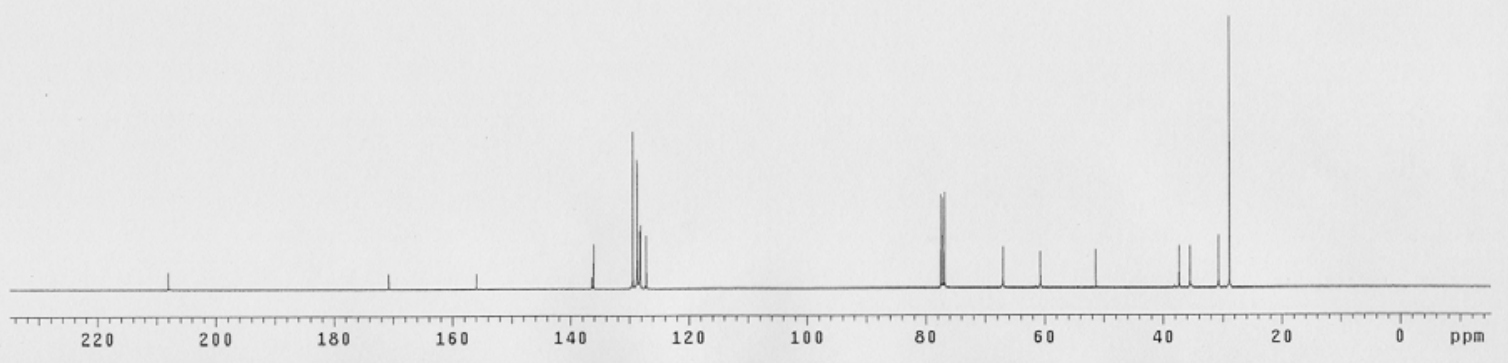

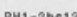

Archive directory: /export/hone/auto/vnnesys/dato
Sanple directory:

Pulse sequence: s2pul

Solvent: CDC13 1 so

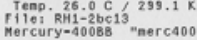

Relax. de lay 1.000 sec

Acain $25188.19 \mathrm{~Hz}$

OSSERVE C13, 100.6982094 RHZ

Power 36 do
continuous
con on

cont inuous 1y on

Line broadening $1.0 \mathrm{~Hz}$

rotai tine $19 \mathrm{gin}, 32 \mathrm{sec}$
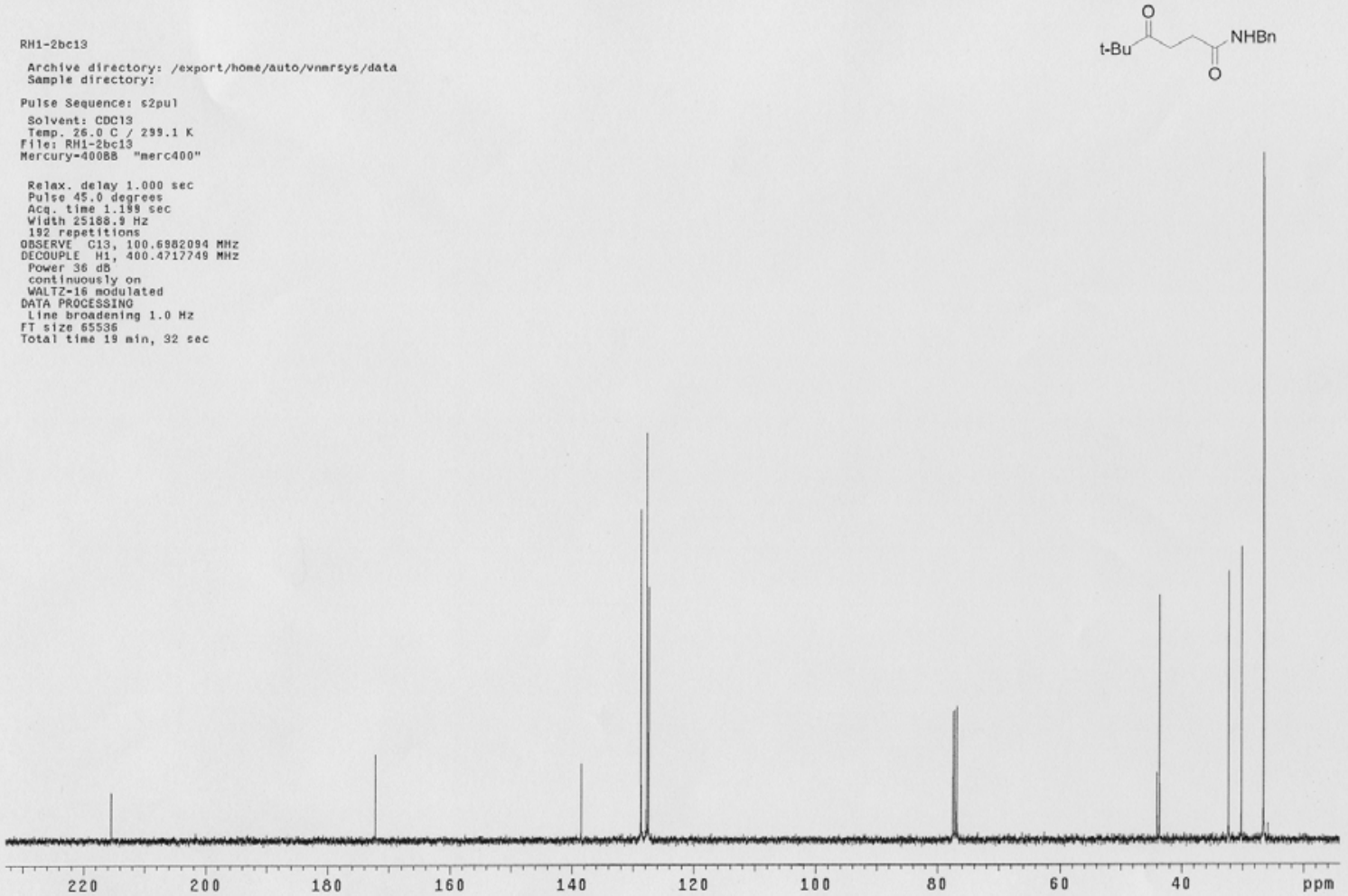
RH1-12C13

Archive directory: /export/home/auto/vnmrsys/date
Sample directory:



Puise Sequence: s2pur

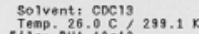

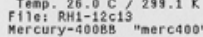

Re lax. de lay 1.000 sec

Acq. time

1000 repetitions
OBSERE C 13 , 100.6982047 RHI
DECOUPLE H1; 400.4717749 KHI

Power 36 de
continuous $1 y$ on
wallz $2-15$

作

FT 512 ize 655366 in

Jk exp27

Archive directory: /export/home/auto/vnarsys/data

Pulse sequence: $52 \mathrm{p}$

Pulse sequence: s2put
Solvent: CoC13 $1399.1 \%$

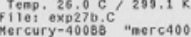

$\overbrace{}^{\circ}$

Relax. dolay $1.000 \mathrm{sec}$

Acg. ${ }^{2}$ ine 1.1999

512 repetitions.
OBgERVE CC13, $100.6981949, \mathrm{KHz}$

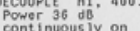

WALt T-16 modu lated
OATA PROCESSTHS

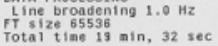

180

160

140

120

100

80

6

ppm

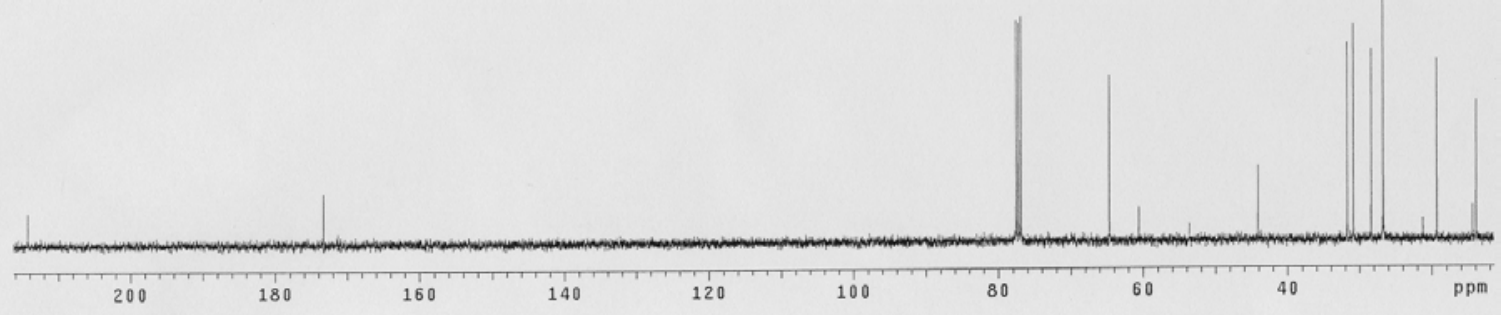



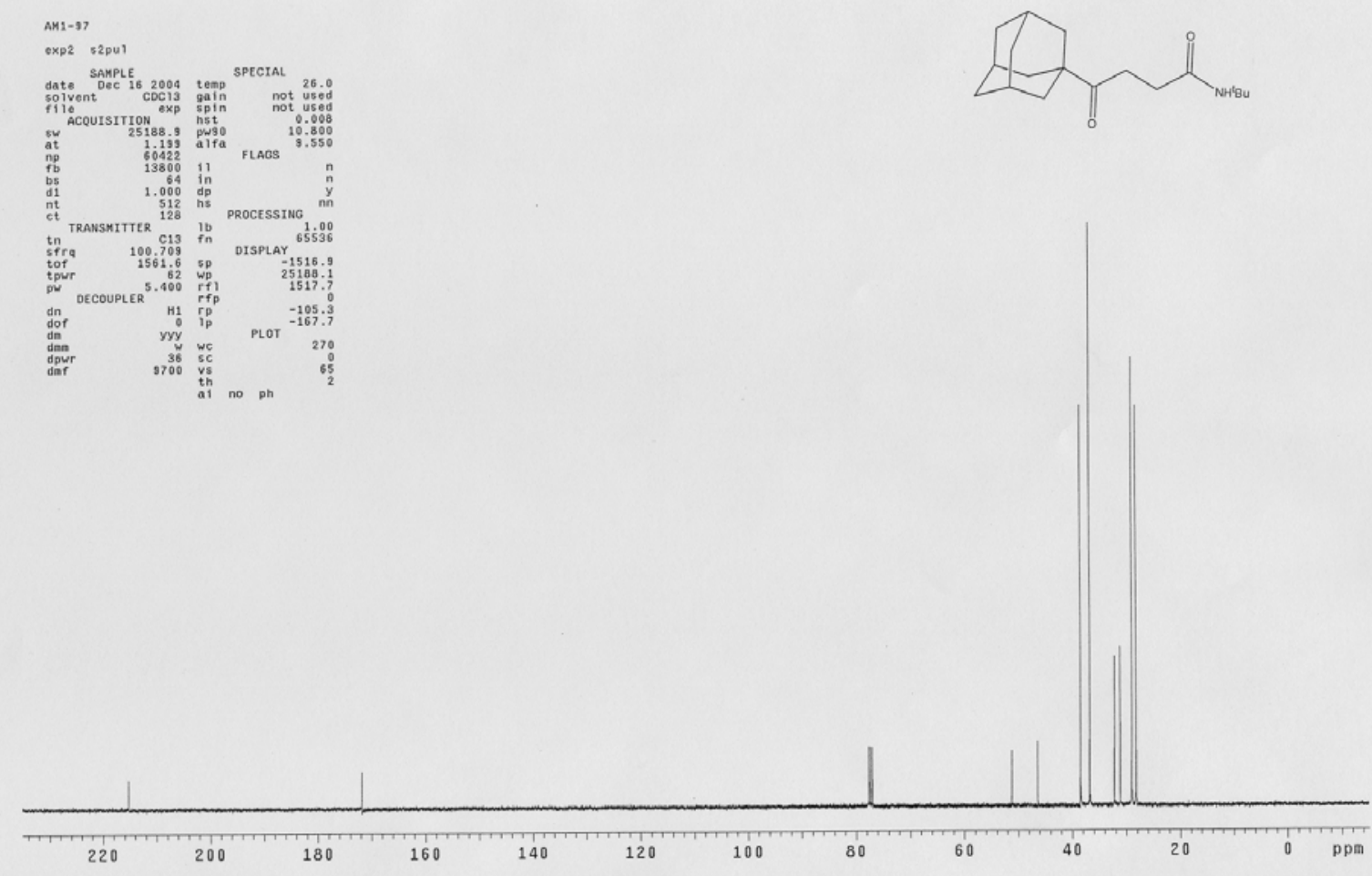

jK exp28b2

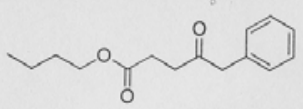

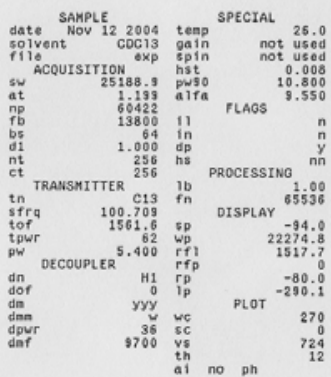

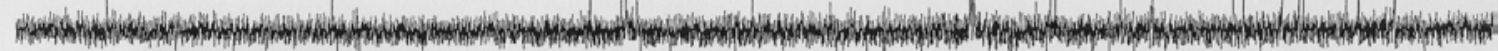

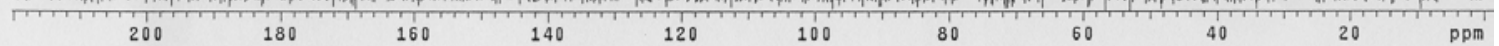




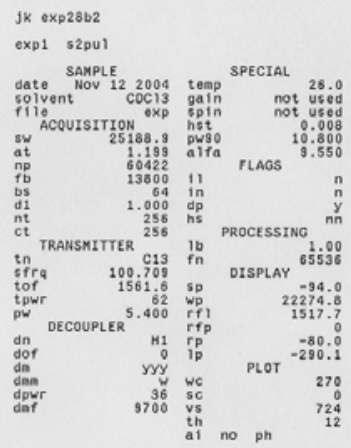

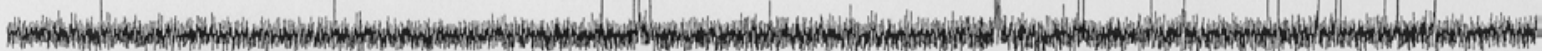
$200 \quad 180 \quad 160$

$160 \quad 140$ 120 100 80

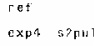

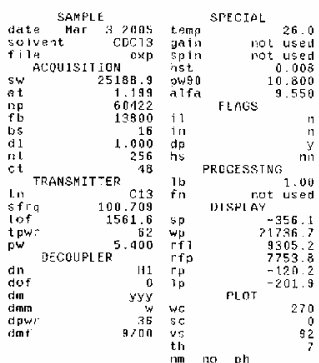
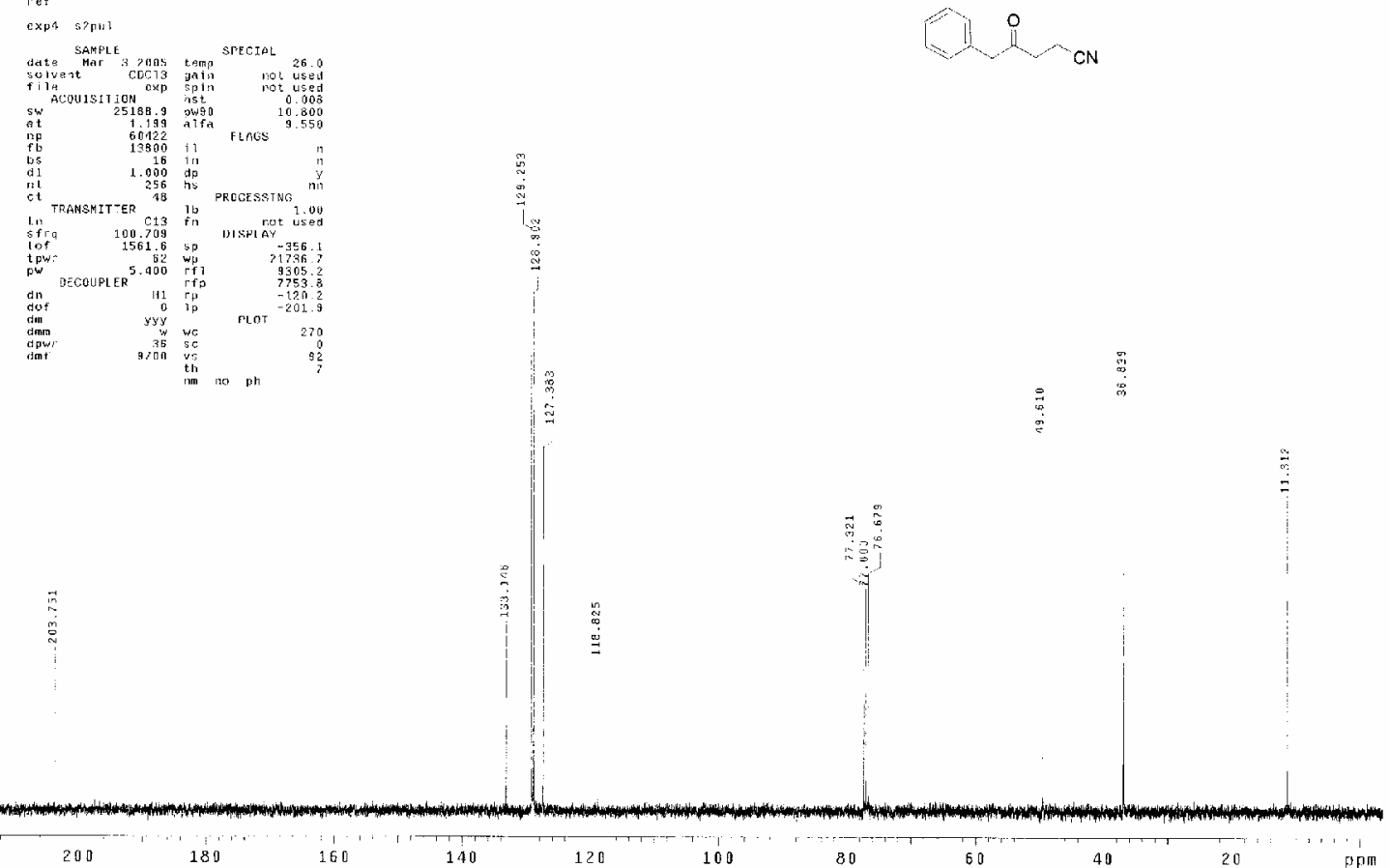
cyr

exp7 s2pul

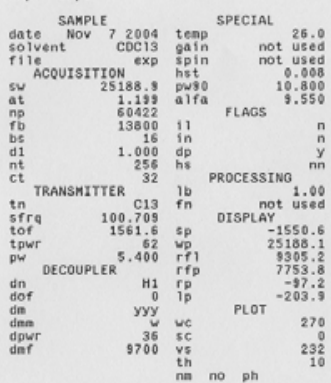

FHlename: AuC

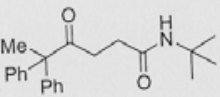



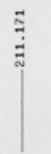
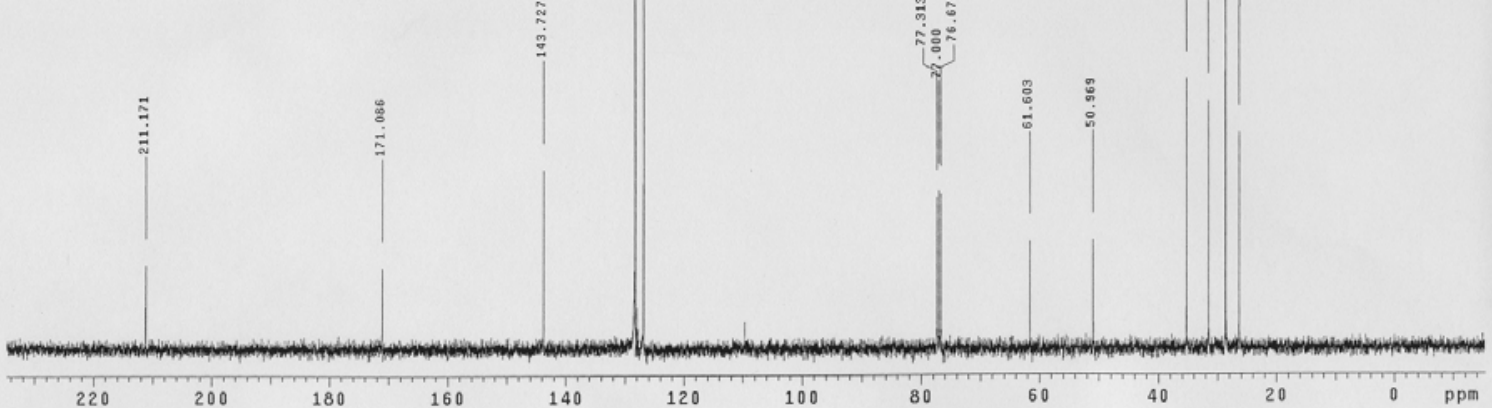

F11ename: AuC

exp2 s2pui

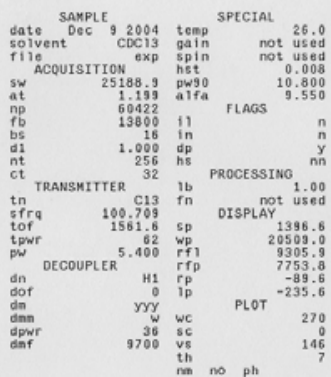

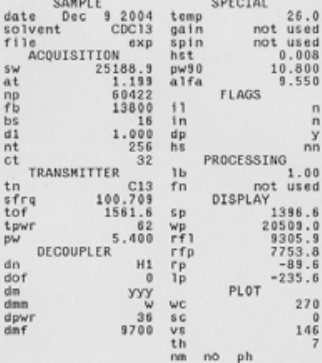

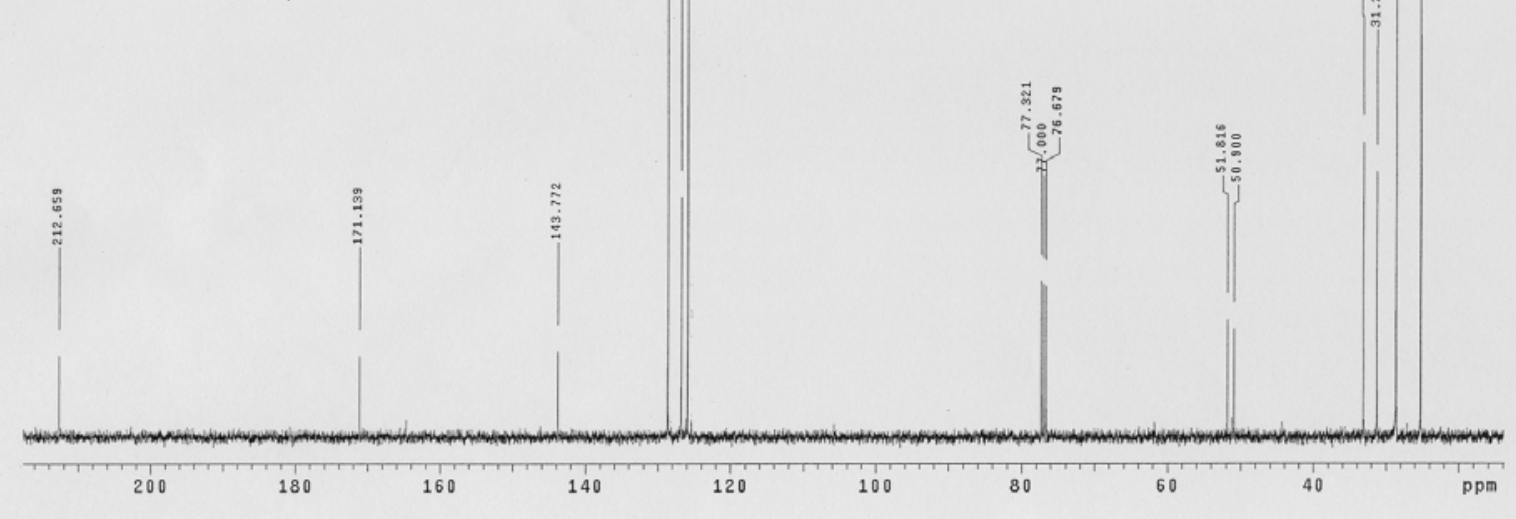

